\title{
The Medial Nucleus of the Trapezoid Body in the Gerbil Is More Than a Relay: Comparison of Pre- and Postsynaptic Activity
}

\author{
Cornelia Kopp-Scheinpflug, ${ }^{1}$ William R. Lippe, ${ }^{2}$ Gerd J. Dörrscheidt, ${ }^{3}$ And Rudolf Rübsamen ${ }^{1}$ \\ ${ }^{1}$ Department of Neurobiology, University of Leipzig, Leipzig, Germany \\ ${ }^{2}$ VMB Hearing Research Center and Oto-HNS, University of Washington, Seattle, WA 98195-7923, USA \\ ${ }^{3}$ Department of General Zoology and Neurobiology, Ruhr-University, Bochum, Germany
}

Received: 26 March 2001; Accepted: 27 April 2002; Online publication: 8 July 2002

\section{ABSTRACT}

The medial nucleus of the trapezoid body (MNTB) plays an important role in the processing of interaural intensity differences, a feature that is critical for the localization of sound sources. It is generally believed that the MNTB functions primarily as a passive relay in converting excitatory input originating from the contralateral cochlear nucleus $(\mathrm{CN})$ into an inhibitory input to the ipsilateral lateral superior olive. However, studies showing that the MNTB itself is also the target of inhibitory input suggest that the MNTB may serve more than a sign-converting function. To examine the fidelity of signal transmission at the CN-MNTB synapse, presynaptic calyceal potentials ("prepotentials"), reflecting the excitatory input to the MNTB neuron, and postsynaptic action potentials were simultaneously monitored with the same electrode during in vivo extracellular recordings from the gerbil's MNTB. Presynaptic activity differed from postsynaptic activity in several respects: (1) Spontaneous and sound-evoked discharge rates were greater presynaptically than postsynaptically. (2) Frequency tuning was sharper postsynaptically than presynaptically. (3) Calyceal terminals and MNTB neurons both showed phasic-tonic response patterns to tonal stimulation, but the duration of the onset response and the level of the tonic component were reduced

Correspondence to: Rudolf Rübsamen, • Department of Neurobiology • University of Leipzig - Talstrasse 33 - 04103 Leipzig, Germany. Telephone: 49-341-9736723; fax: 49-341-9736848; email: rueb@ rz.uni-leipzig.de postsynaptically. (4) Phase-locking to sound frequencies up to $1 \mathrm{kHz}$ was greater postsynaptically than presynaptically. (5) The rate-intensity characteristics of pre- and postsynaptic activities differed significantly from each other in half of the MNTB neurons. To test the hypothesis that acoustically evoked inhibition of MNTB neurons contributed to the relatively lower levels of postsynaptic discharge, two-tone stimulation was applied, wherein the response to one tone-burst, set at the neuron's characteristic frequency, can be reduced by addition of a second "inhibitory" tone. The inhibitory tone caused a much larger reduction in post- than in presynaptic activity, indicating an acoustically evoked inhibitory influence directly on MNTB units. These findings show that transmission at the CN-MNTB synapse does not occur in a fixed one-to-one manner and that the response of MNTB neurons reflects the integration of their excitatory and inhibitory inputs.

Keywords: MNTB, calyx of Held, prepotential, auditory brainstem, neuronal inhibition, two-tone stimulation

\section{INTRODUCTION}

The superior olivary complex (SOC) is composed of several different nuclei that contribute to the processing of interaural differences in time (ITDs) and intensity (IIDs), both key features for the localization of sound sources. Neurons of the medial nucleus of 
the trapezoid body (MNTB) receive excitatory input through large presynaptic terminals, the calyces of Held, from globular bushy cells located in the contralateral anteroventral cochlear nucleus (AVCN) (Smith et al. 1991). The activity of these large presynaptic endings can be monitored in extracellular single-unit recordings as discrete "prepotentials" that precede the postsynaptic spikes (Guinan and $\mathrm{Li}$ 1990). MNTB neurons, in turn, form inhibitory synapses onto neurons of the lateral superior olive (LSO). By comparing this inhibitory input with excitatory input, they receive from the ipsilateral cochlear nucleus $(\mathrm{CN})$, LSO neurons encode the relative intensity of sound at the two ears.

According to the current model of IID processing, the MNTB functions as a "passive relay" to convert the excitatory input originating from the contralateral CN into an inhibitory output to the LSO (Irvine 1986; Sommer et al. 1993). That is, aside from the sign conversion that occurs at the MNTB-LSO synapse, it is generally believed that the spike output of MNTB neurons is in all respects an exact duplicate of its afferent excitatory input. However, recent immunocytochemical and electrophysiological studies showing that the MNTB itself is also the target of inhibitory inputs suggest that this model of MNTB functioning needs to be reconsidered. Inhibitory (noncalyceal) GABAergic and glycinergic boutons on the somata of MNTB neurons have been demonstrated by immunocytochemical labeling (Roberts and Ribak 1987; Adams and Mugnaini 1990). Transmission electron microscopic studies corroborated these findings by showing that these small terminals enclose flattened vesicles, which are thought to contain inhibitory transmitters (Cant 1991; Osen and Ottersen 1996). The GABAergic terminals may originate from neurons in the ventral nucleus of the trapezoid body (Ostapoff et al. 1997), which themselves receive excitatory input from axon collaterals of globular bushy cells (Kuwabara et al. 1991). The superior paraolivary nucleus, in which most neurons label for GABA, might provide another source of GABAergic input to the MNTB (Helfert et al. 1989). The glycinergic terminals arise, in part, from recurrent projections of MNTB neurons (Kuwabara and Zook 1991; Smith et al. 1998). To date, the physiological effect of inhibitory neurotransmitters and of inhibitory input to the MNTB has been studied exclusively using slice preparations (Banks and Smith 1992; Forsythe and Barnes-Davies 1993b; Wu and Kelly 1995; Turecek and Trussell 2001). Electrical stimulation of the trapezoid body in rat brain slices gives rise to IPSPs in MNTB neurons, which can be blocked by strychnine (Banks and Smith 1992; Forsythe and Barnes-Davies 1993b). In addition, the membrane resistance of MNTB cells in the mouse is lowered by application of GABA and glycine (Wu and Kelly 1995).

The innervation of MNTB neurons by extrinsic and intrinsic inhibitory afferents, the effect of inhibitory neurotransmitters, and the fact that the MNTB projects to several other brainstem auditory nuclei in addition to the LSO (Kuwabara and Zook 1991; Schofield and Cant 1991; Banks and Smith 1992; Sommer et al. 1993; Schofield 1994; Smith et al. 1998) suggest that the MNTB does not serve only as a sign-converting relay to the LSO. To test this hypothesis, the excitatory afferent input to the MNTB neuron and the firing of the MNTB neuron itself were simultaneously monitored with the same electrode during in vivo extracellular recordings in the gerbil. The results show that the postsynaptic response properties of MNTB neurons differ in several respects from those of their excitatory afferents, these differences being due, in part, to inhibitory influences on the MNTB neurons. Portions of these results were previously reported in abstract form (Kopp et al. 1997, 1998).

\section{METHODS}

\section{Animals and animal care}

The experiments were performed at the Neurobiology Laboratories of the Zoological Department of the University of Leipzig (Germany). All experimental procedures were approved by the Saxonian District Government, Leipzig. Adult pigmented (agouti) Mongolian gerbils (Meriones unguiculatus), aged 3-6 months and weighting 50-80 g, were used in the experiments. The animals were obtained from the animal care facilities of the Zoological Department of the University of Leipzig.

\section{Surgical preparation}

During the experiments and the surgical preparation, the animals were anesthetized with an initial dose of $0.3 \mathrm{~mL} / 100 \mathrm{~g}$ body weight of a 10:1 mixture of ketamine hydrochloride $(0.13 \mathrm{mg} / \mathrm{g}$ body weight; Parke-Davis) and xyaline hydrochloride $(0.005 \mathrm{mg} / \mathrm{g}$ body weight; Bayer). During the recording experiments, a constant level of anesthesia was maintained by hourly injections of one-third of the initial dose. The skull of the experimental animal was exposed along the dorsal midsagittal line, and a small metal bolt for supporting the animal in the stereotaxic recording device was glued to the bone overlaying the forebrain. Two 500- $\mu \mathrm{m}$-diameter holes were drilled in the skull 2000-2300 $\mu \mathrm{m}$ caudal to the lambda suture, which correspond to positions above the rostral third of the cerebellum. The first drill hole, located 1500 
$\mu \mathrm{m}$ lateral to the midline, was used to position the reference electrode in the superficial cerebellum. Through the second drill hole located over the midline, the recording electrode was inserted at an angle of $5^{\circ}-8^{\circ}$ to the midsagittal plane.

\section{Acoustic stimulation}

All acoustic stimuli were digitally generated with 16bit accuracy by a PC486/33 computer. The stimuli were delivered at 250 kilosamples/s per channel through a two-channel, 14-bit D/A converter including a custom-made low-pass resynthesis filter $(50 \mathrm{kHz}$ cutoff), and a software-controlled attenuator (0-120 $\mathrm{dB}$ in 1-dB steps). A source selector allowed the two output signals to be directed either separately or concurrently to the two channels of a stereo amplifier (Microline) that drove the sound transducers. Custom-made acoustic transducers were designed for "near field" stimulation of the ear. Sound was delivered through a plastic tube $(5-\mathrm{mm}$ diameter at the end) inserted into the outer ear canal and positioned approximately $5 \mathrm{~mm}$ from the animal's eardrum. The frequency characteristic of the coupler was measured with a 0.25 -in. condenser microphone (Bruel \& Kjaer type 2619) coupled to a short plastic tube mimicking the conditions in the ear canal. A computer-controlled procedure determined for 50 frequencies per decade the sound pressure levels at a defined input voltage. The data were stored in a computer file that was used during experiments for online level correction of stimulus intensities.

\section{Data collection and analysis}

All recording experiments were performed in a sound-attenuated chamber (Industrial Acoustics, Type 400). Generally, each animal was used in 4-5 daily recording sessions over the period of a week, with each recording session lasting 5-6 hours. During the experiments the body temperature was kept between $36^{\circ} \mathrm{C}$ and $37.5^{\circ} \mathrm{C}$ by positioning the animal on a temperature-controlled heating pad and maintaining the temperature of the recording chamber at 28$30^{\circ} \mathrm{C}$. After daily recording sessions, the electrodes were removed and the holes in the skull were reversibly closed with a clot of agarose.

Multiunit mapping. The tonotopic organization of the medial nucleus of the trapezoid body of the gerbil is comparable to that of other mammals (cat: Guinan 1972b; rat: Sommer et al. 1993; Smith et al. 1998). Differentiating the MNTB from other nuclei within the SOC during recording is facilitated by the fact that the MNTB contains only monaural units, i.e., they are all acoustically driven only by the contralateral ear. In the first recording session for each ani- mal, the stereotaxic coordinates of the MNTB were determined by online analysis of acoustically evoked multiunit activity. For this, glass micropipettes (Clark Electromedical Instruments) filled with $3 \mathrm{M} \mathrm{KC1}$ and having impedances of 5-10 M $\Omega$ were used. Employing monaural and binaural tone-burst stimulation, the characteristic frequencies (CF) of the multiunits were measured every $100 \mu \mathrm{m}$ in several penetrations in the estimated position of the MNTB (test range 0.1-60 kHz and 0-90 dB SPL). The MNTB, located ventrally in the brainstem just lateral to the midline, was typically reached at a penetration depth of 7000$8000 \mu \mathrm{m}$, depending on the size of the animal. Evaluation of the distribution of the units' CFs along the mediolateral nuclear extent allowed the medial and lateral borders of the MNTB to be demarcated physiologically.

Single-unit recordings. Following multiunit mapping, recordings from single units were made through glass micropipettes (Clark Electromedical Instruments) pulled to a tip diameter of less than $1 \mu \mathrm{m}$ and filled with $3 \mathrm{M}$ KC1. Best signal-to-noise ratios and the highest probability of being able to simultaneously record prepotentials and action potentials were obtained with electrode impedances of 15-30 M $\Omega$. The activity of isolated single units was bandpass filtered $(300 \mathrm{~Hz}-10 \mathrm{kHz})$ and amplified to the voltage range of the spike discriminator and of the $\mathrm{A} / \mathrm{D}$ converter. Single units were identified by three criteria: (i) relatively constant spike height, (ii) constant waveform, and (iii) large signal-to-noise ratio $(>2)$. For acquisition of the time of occurrence of pre- and postsynaptic events, the amplified recording signal was delivered to a custom-made window discriminator followed by an event-timer PC interface. During a single recording, the window discriminator was set to be triggered by either the prepotentials or by the postsynaptic action potentials using a combined slope, level, and dead-time criterion (Fig. 4A). This allowed the two types of signals to be differentiated for analysis. Events were acquired with $100 \mu$ s resolution using custom-written real-time computer programs. Waveforms were recorded with an A/D conversion rate of $20,000 / \mathrm{s}$ ( $50 \mu$ s sample interval).

Electrophysiological data were acquired using the following protocol: First, the excitatory response areas for the action potentials and the prepotentials were separately measured by random presentation of pure-tone pulses $(100 \mathrm{~ms}$ duration, $5 \mathrm{~ms}$ rise-fall time) within a given matrix of $16 \times 15$ frequency/ intensity pairs (240 combinations). Each frequency/ intensity combination was presented five times in a predefined frequency/intensity array (Fig. 1A). The timing of the spikes and the number of spikes were measured during the 100 -ms period of stimulus presentation and in the absence of acoustic stimulation 

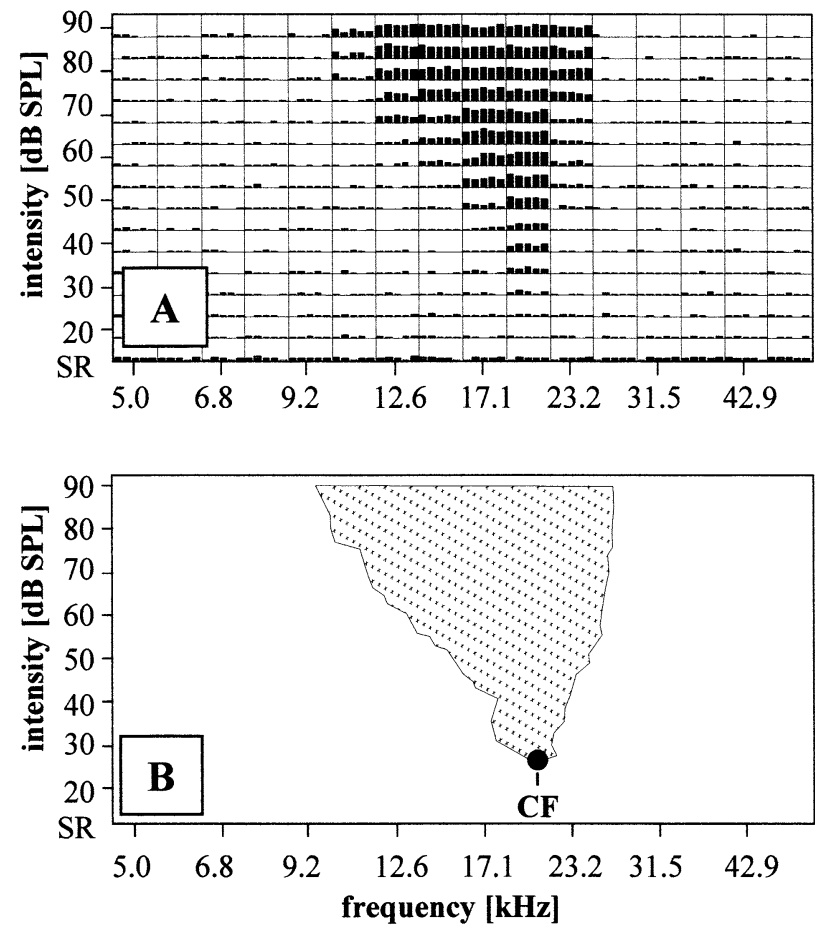

FIG. 1. Acquisition of excitatory frequency/intensity response areas (example: unit 86-0102). Sixteen discrete pure-tone stimuli (here frequencies between 5.0 and $50 \mathrm{kHz}$ ) were presented at 15 different intensities between 20 and $90 \mathrm{~dB}$ SPL, and as a mute stimulus (bottom row). Tone bursts (100 ms stimulus duration, $100 \mathrm{~ms}$ interstimulus interval) were presented in five blocks in which all 240 frequency/intensity combinations were tested in an individual random order. A. The spike rate evoked by each respective stimulus is indicated by the height of the bars (log scale, 40/s max). Spontaneous activity is shown in the bottom row (SR). Tone-evoked discharges were found in a frequency/intensity range with a typical narrowing of the excitatory frequency range towards lower stimulus levels. B. Frequency-threshold curve enclosing the excitatory response area calculated from the response area shown in $\mathrm{A}(\mathrm{CF}=19.9 \mathrm{kHz}$; threshold $=25 \mathrm{~dB}$ SPL).

(spontaneous activity). From these data the CF, threshold, $Q$ value (bandwidth of excitation/CF), and rate-level function (RLF) were calculated.

Verification of recording sites. In each animal, all recording sites were verified histologically using HRP marking. At the end of the last recording session, the recording electrode was replaced with a glass micropipette filled with a solution of $9 \% \mathrm{HRP}$ in $0.9 \% \mathrm{NaCl}$, which was injected microiontophoretically ( $2 \mu \mathrm{A}$ for 5 min) into the recording site. The position of the HRP electrode was confirmed by measuring the $\mathrm{CF}$, threshold, and temporal response pattern of the neuronal activity at the injection site. The animal was allowed to survive for 24 hours and was then perfused via the left ventricle with $0.9 \% \mathrm{NaCl}$ solution followed by fixative $(2.5 \%$ paraformaldehyde in $0.1 \mathrm{M}$ phosphate buffer, $\mathrm{pH}$ 7.4) for 20-25 min. The brain was cut on a vibratome and the tissue sections $(100 \mu \mathrm{m})$ were reacted using the 3,3'-diaminobenzidine (DAB) reaction to visualize the HRP mark (Adams 1981). Following staining with cresyl violet, the tissue sections were examined under the light microscope and the electrode tracks and recording sites were reconstructed.

Analysis of spike recordings. Besides methods for visualizing spike responses (poststimulus time histogram [PSTH], spike counts/rates, dot display), we also computed synchronization indices, rate-level functions, and frequency-threshold curves. Typical electrophysiological recording data from MNTB neurons are shown in Figure 1A. In this figure the height of each bar represents the number of spikes (logarithmic scale, maximum 40 spikes) during a single stimulus presentation of the given frequency and intensity combination. The neuronal response area shown in Figure 1B was defined by the range of frequency/intensity combinations that evoked discharge rates equal to or greater than the $90 \%$ confidence level above spontaneous activity. The outline of the response area defined the unit's frequencythreshold curve (FTC), from which the CF and the $\mathrm{CF}$ threshold were determined (Fig. 1B).

Statistical analyses of the data were performed with SigmaStat/SigmaPlot ${ }^{\mathrm{TM}}$ (Jandel/SPSS). For normally distributed data, results are expressed as means \pm standard deviation and statistical significance was assessed using the Student's paired $t$-test. For other distributions, results are expressed as medians [25\%, 75\% quartiles], and significance was assessed using the Wilcoxon signed rank test or the Mann-Whitney rank sum test.

Waveform analysis. The number and kind of different waveforms in an extracellular recording can be determined by the means of the principal component analysis (PCA), followed by cluster analysis. First, Abeles and Goldstein (1977) applied this technique to extract the activity of individual units from a multiunit recording. In contrast, we used it for separating isolated prepotentials (IPP) from pre\&action potential compounds in recordings from single units in the MNTB. In our application, all waveforms (2.5-ms time epochs) that met a user-defined trigger criterion comprising upper and lower thresholds, slope direction, and dead time were selected from digitized waveform recordings (Fig. 4B). Prior to PCA, the selected signals were multiplied by a hamming-like windowing function to enhance the relative weight of the center region. PCA then describes each waveform from this ensemble as the weighted sum of a number of basic waveforms, the principal components (PCs). Our MATLAB $5{ }^{\circledR}$ implementation of the PCA, i.e., the evaluation of the principal components and of the weights associated with the individual time course, follows the Karhunen-Loeve expansion (Glaser and Ruchkin 1976). Goodness of fit is expressed as the 
A) $84-0302$

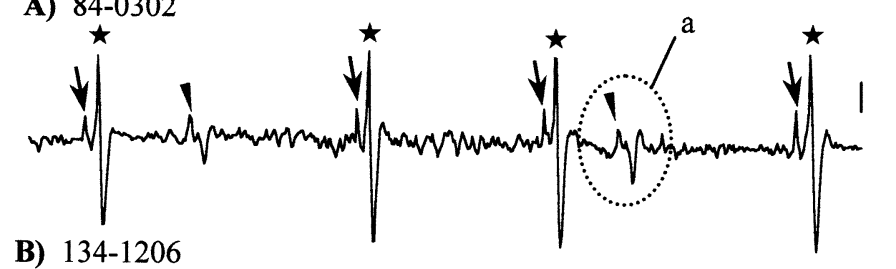

B) $134-1206$

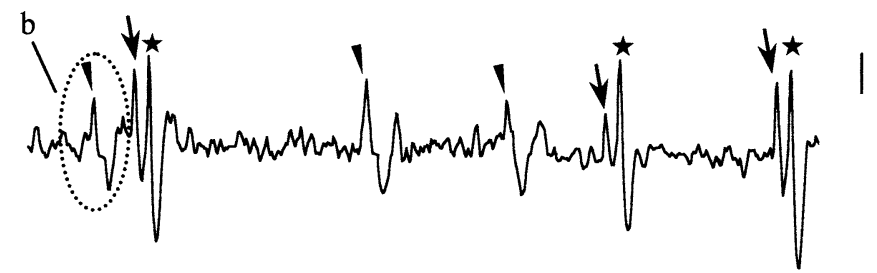

C) $134-0215$

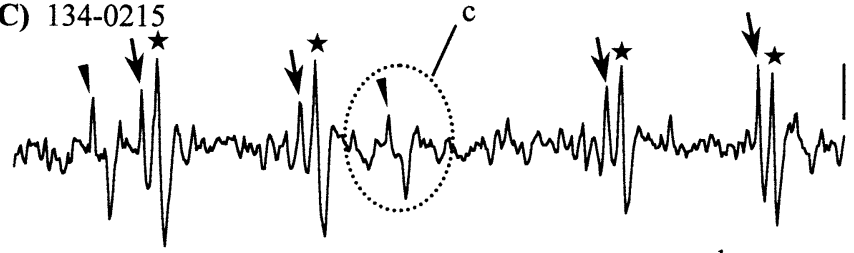

D) $65-0303$

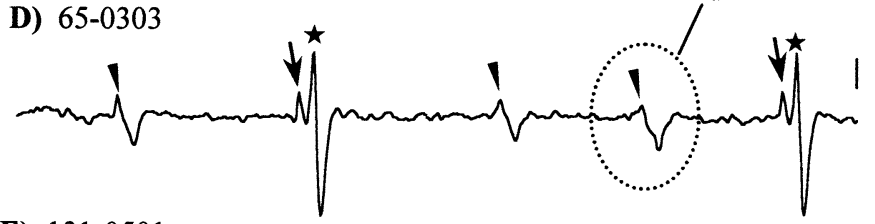

E) $131-0501$

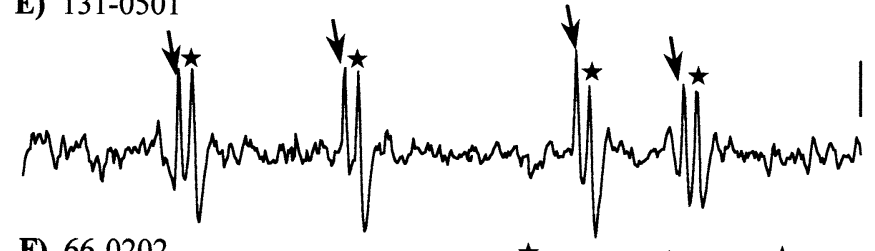

F) $66-0202$

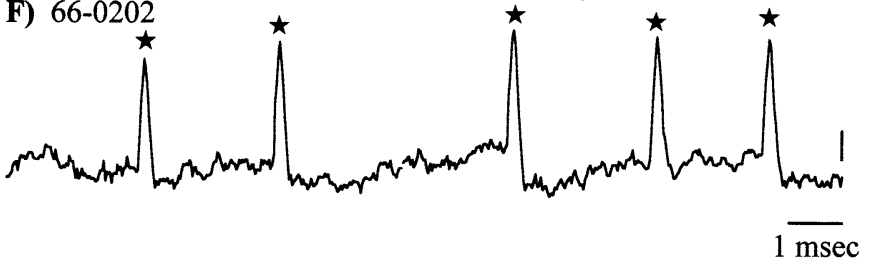

a<smiles>CCCCC(C)CCC</smiles>

$0.5 \mathrm{msec}$
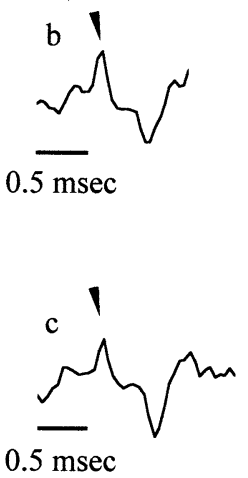

d

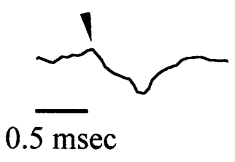

$0.5 \mathrm{msec}$
FIG. 2. Waveforms of extracellularly recorded action potentials of six MNTB units. Characteristic frequencies were (A) $27.0 \mathrm{kHz}$, (B) $10.9 \mathrm{kHz}$, (C) $6.5 \mathrm{kHz}$, (D) $9.5 \mathrm{kHz}$, (E) $17.0 \mathrm{kHz}$, (F) $1.1 \mathrm{kHz}$. A-E. Units in which action potentials (stars) were preceded by prepotentials (arrows). In A-D, additionally isolated prepotentials (IPPs, arrow heads) occur that are not immediately followed by action potentials. Note that in the IPPs the initial positive signal component is followed by a somewhat delayed negativity. The delay between the positive and the negative components becomes more prominent when the time scale is enlarged (circled signals and a-d). C, D. Two examples in which the prepotential amplitude in part surpasses the action potential amplitude. $\mathbf{F}$. For comparison, a unit is shown which lacks prepotentials. Horizontal scale bar $=1 \mathrm{~ms}$ (for all units), vertical scale bars $=0.2 \mathrm{mV}$. quotient of (variance of the approximating set of waveforms)/(variance of the original ensemble of waveforms), so that $100 \%$ indicates perfect approximation. Depending on the variance of the ensemble, only a few PCs and a correspondingly small number of weights may give a sufficient description of the great majority of the original waveforms. In our cases, two seemed to be enough (see Results, especially Fig. 4D-G).

\section{RESULTS}

Waveform of neuronal discharges

Extracellular recordings were made from a total of 146 neurons in the MNTB of 23 Mongolian gerbils.
The discharges of $58 \%(85 / 146)$ of the neurons showed complex waveforms that were comparable to those described previously for the cat MNTB (Guinan 1972a; Guinan and Li 1990). Fifty-four of these socalled "prepotential units" provided the quantitative data for the findings described below. The discharges of prepotential units consisted of bipolar action potentials preceded immediately by prepotentials (Fig. 2A-E). The prepotentials preceded the action potentials by $0.46 \pm 0.12 \mathrm{~ms}$ ( $n=15$ units). These signal complexes, consisting of an action potential and an immediately preceding prepotential, are termed pre\&action potential compounds. In between prepotential units and intermixed with them, other units were recorded that either lacked prepotentials (Fig. $2 \mathrm{~F}$ ) or had prepotentials that were too small in am- 
plitude to be reliably distinguished from the noise floor $(61 / 146=42 \%$ of the entire ensemble $)$. Because such units were recorded in between prepotential units, we believe that these recordings probably reflect MNTB activity recorded with an "unfavorable" spatial relation between the tip of the electrode and the calyx terminal. For the prepotential units, the most stable recordings, those allowing recording times of up to 1 hour and more, occurred when the prepotentials were approximately one-third the size of the postsynaptic spikes (prepotential: $0.46 \pm 0.17 \mathrm{mV}$ vs. action potential: $1.2 \pm 0.4 \mathrm{mV}$; $n=15$ units).

As would be expected from the nature of extracellular recording, the amplitudes of the action potentials and prepotentials differed among units and sometimes during the course of recording from the same unit (Fig. 2A-E). The variability in signal amplitude was particularly noticeable in recordings with relatively large variations in baseline activity (e.g., Fig. 2B, C). Similarly, the amplitude relation between prepotentials and action potentials varied, particularly with small changes in the electrode position. This was observed during most recordings. In the one example shown in Figure 3, advancing the electrode by $7 \mu \mathrm{m}$ caused the amplitude of the prepotentials to increase and become larger than the postsynaptic action potentials.

In many recordings from prepotential units, a second type of signal occurred in between the pre\&action potential compounds. By the shape of their positive onset components, these signals resembled prepotentials but were not followed immediately by spike discharges. These isolated small-sized signals recorded in the present study will be referred to as isolated prepotentials (IPPs). Instead of following spike discharges, negativities occurred at the position of the "missing" action potentials (Fig. 2A-D). These negativities followed the (positive) IPPs with a 0.40.5 -ms delay (maximum amplitude). The negativities are thought to indicate extracellularly recorded excitatory postsynaptic potentials (EPSPs; see Discussion for further consideration on this topic). Guinan and $\mathrm{Li}$ (1990) previously reported occasional "spike failures" in their recordings from the cat MNTB. These were small-sized signals that, as in our own recordings, also lacked immediately following action potentials. However, Guinan and Li did not subject these signals to a more detailed analysis. In the present study, the terms prepotential or presynaptic activity are used when referring jointly to the spike preceding prepotentials (SPPs) in the pre\&action potential compounds and to the IPPs in the IPPEPSP complexes. The term postsynaptic activity is used to refer to the spike discharge of the MNTB neuron.

\section{A) $8074 \mu \mathrm{m}$}

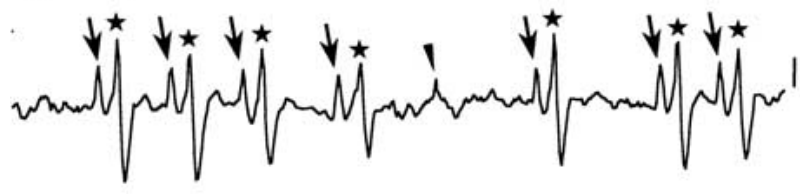

B) $8077 \mu \mathrm{m}$
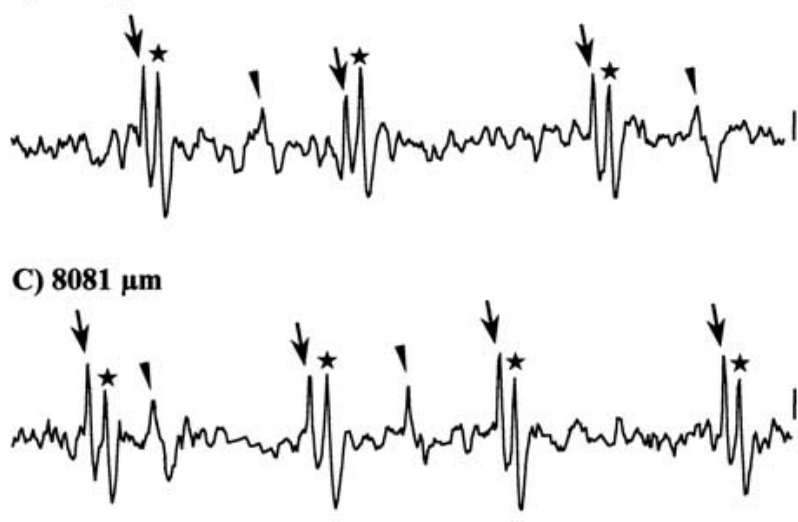

D)

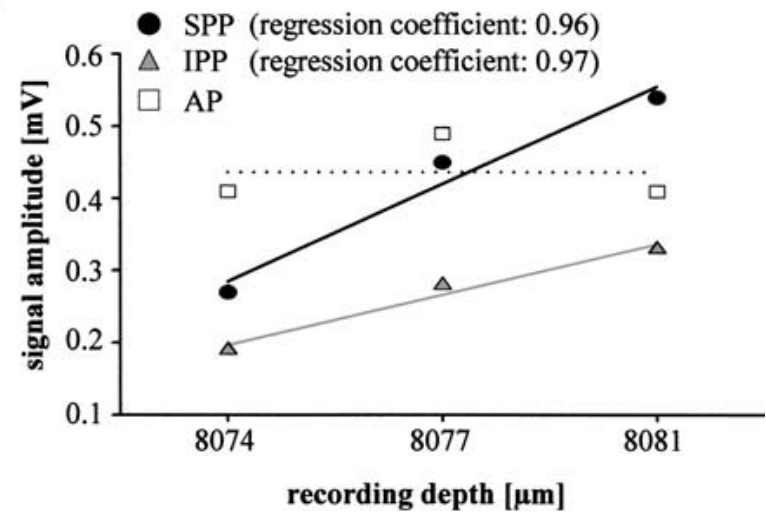

FIG. 3. Differences in relative amplitudes of spike preceding prepotentials (SPPs; arrows) and postsynaptic action potentials (stars) of a single unit acquired with slightly altered electrode positions. A. SPP amplitudes reach one-third to one-half of action potential amplitudes. B. Advancing the electrode by $3 \mu \mathrm{m}$ caused the SPP amplitudes to rise to about the same height as the action potentials. C. Further movement of the electrode by another $4 \mu \mathrm{m}$ caused the SPP amplitudes to surpass the action potential amplitudes. Note that the amplitudes of the isolated prepotentials (IPPs; arrowheads) also tend to increase in amplitude with protraction of the electrode. Time scale $(1 \mathrm{~ms})$ and amplitude scale $(0.2 \mathrm{mV})$ are the same in A-C. D. Consistent increase of SPP amplitudes and IPP amplitudes.

For the interpretation of the analyses performed in the present study, the origin and nature of the IPPs is critical. Based upon the findings presented below, we believe that all IPPs in the recordings from a given MNTB unit reflect the discharge of the same neural element. Furthermore, we suggest that the neural element generating the IPPs is the same presynaptic calyx terminal from which SPPs also arise. Thus, we interpret IPPs to be calyceal discharges (prepotentials) that have failed to evoke a postsynaptic spike. The alternative possibility-that our recordings were 
of the multiunit type-seems remote, given the small tip size and high impedance (15-25 $\mathrm{M} \Omega$ ) of the recording electrodes used. Also, the interdependence of PSTH types of IPPs and pre\&action potential compounds provides very compelling evidence that our recordings were not of the multiunit type (presented below in Figs. 13 and 14).

If all the IPPs in a given recording are generated by the same neural element, then the recording should be composed of exactly two different types of waveforms: the pre\&action potential compound and the IPP. The possibility of multiunit recording, which would mean that IPPs reflect the discharge of different neural units, e.g., several neurons or fibers of passage, predicts that more than two waveforms could be seen. In order to objectively determine the number of independent waveforms, the PCA was performed on digitized waveform recordings of 30 prepotential units. Typically, this analysis is used to separate different units in a multiunit recording. However, here we use the PCA to separate isolated prepotentials from pre\&action potential compounds in recordings from single prepotential units. The results of the PCA on one such prepotential unit are shown in Figure 4. The trigger level was set to detect all prepotential signals, both IPPs and SPPs, and a dead time of $1 \mathrm{~ms}$ was used to avoid double triggering on the spike discharges themselves (Fig. 4A). The ensemble of 202 superimposed signals upon which the analysis was based is shown in Figure 4B. All of the signals could be described by the first two principal components (Fig. 4C) with a $87 \%$ efficiency, i.e., each of the 202 recorded signals can be adequately explained by the weighted sum of two functions, PC1 and PC2. The analysis of the degree to which PC1 and PC2 contributed to each signal yielded a bimodal distribution as seen from the scatterplot in Figure 4D. The cluster with the higher PC1 weights (cluster 1) represents the pre\&action potential compounds (Fig. $4 \mathrm{~F})$, and the cluster with the lower PC1 weights (cluster 2) identifies the IPPs (Fig. 4G). It should be noted that in both clusters the waveforms contain prepotentials, i.e., prepotentials followed by action potentials in cluster 1 and isolated prepotentials in cluster 2. Figure $4 \mathrm{E}$ shows the resynthesis of the ensemble based solely on PC1 and PC2 and on their corresponding weights. Obviously, already this simple description reflects the subdivision of the waveform ensemble into two, one missing and one containing action potentials. (The major differences between Figure $4 \mathrm{E}$ and the original ensemble in Figure $4 \mathrm{~B}$ are largely explained by the handling of the original waveforms prior to PCA [see Methods]). In summarizing, the results corroborate that only two different types of waveforms were distinguishable in the recording: the pre\&action potential compound and the
IPP. The PCA yielded comparable results for all 30 units, as indicated by the $85 \%-95 \%$ efficiency by which the first two principle components jointly describe the recorded signals.

A number of other observations provide evidence that IPPs and SPPs originate from the same source, namely, the presynaptic calyx: (1) During recording, IPPs and SPPs always appeared and disappeared in concert. Slight advancement of the recording electrode causes coherent changes in SPP and IPP amplitudes (Fig. 3). (2) IPPs were observed only in recordings from prepotential units and never in the recordings from the 61 of 146 MNTB units that lacked immediately preceding prepotentials. Such nonprepotential units could be recorded with the same electrode and in the same penetration in which the prepotential units were recorded. (3) The shapes of the SPPs and the IPPs were very similar, although the IPPs tended to be somewhat smaller in amplitude. (4) SPPs and IPPs had comparable onset slopes and thereby could be selected by the same slope criterion $(0.15 \mathrm{mV} / \mathrm{ms})$.

These different features suggest that the SPPs and the IPPs are generated by the same neuronal element, the calyces of Held. Thus, it is possible to simultaneously monitor with the same extracellular electrode both the discharge activity of the MNTB neuron itself and the neuron's afferent excitatory input (SPPs and IPPs). A differential analysis of the respective activities provides the basis for analyzing the properties of signal transmission at the $\mathrm{CN}-$ MNTB synapse.

\section{COMPARISON BETWEEN PRE- AND POSTSYNAPTIC ACTIVITY}

\section{Spontaneous and sound-evoked activity}

Presynaptic spontaneous discharge rates ranged from 1 to 308 pps (median [25\%, 75\% quartiles] = 49 [17, 79] pps). This was significantly larger than the spontaneous rates measured postsynaptically (0-189 pps; median: $21[6,45]$ pps; Wilcoxon signed rank test; $p \leq 0.001$; Fig. 5). In interpreting these data, it is important to bear in mind that individual postsynaptic action potential discharges were always immediately preceded by a single prepotential. Thus, the relatively lower discharge rates observed postsynaptically reflect a certain degree of "failure" of signal transmission at the $\mathrm{CN}-\mathrm{MNTB}$ synapse, i.e., some prepotentials (IPPs) did not give rise to postsynaptic action potentials. To quantify this, a "failure rate," the percentage of prepotentials that were not immediately followed by an action potential, was calculated for each unit (Fig. 5). The values ranged from $6 \%$ to 95\% (median: 52 [29, 64]\%). 

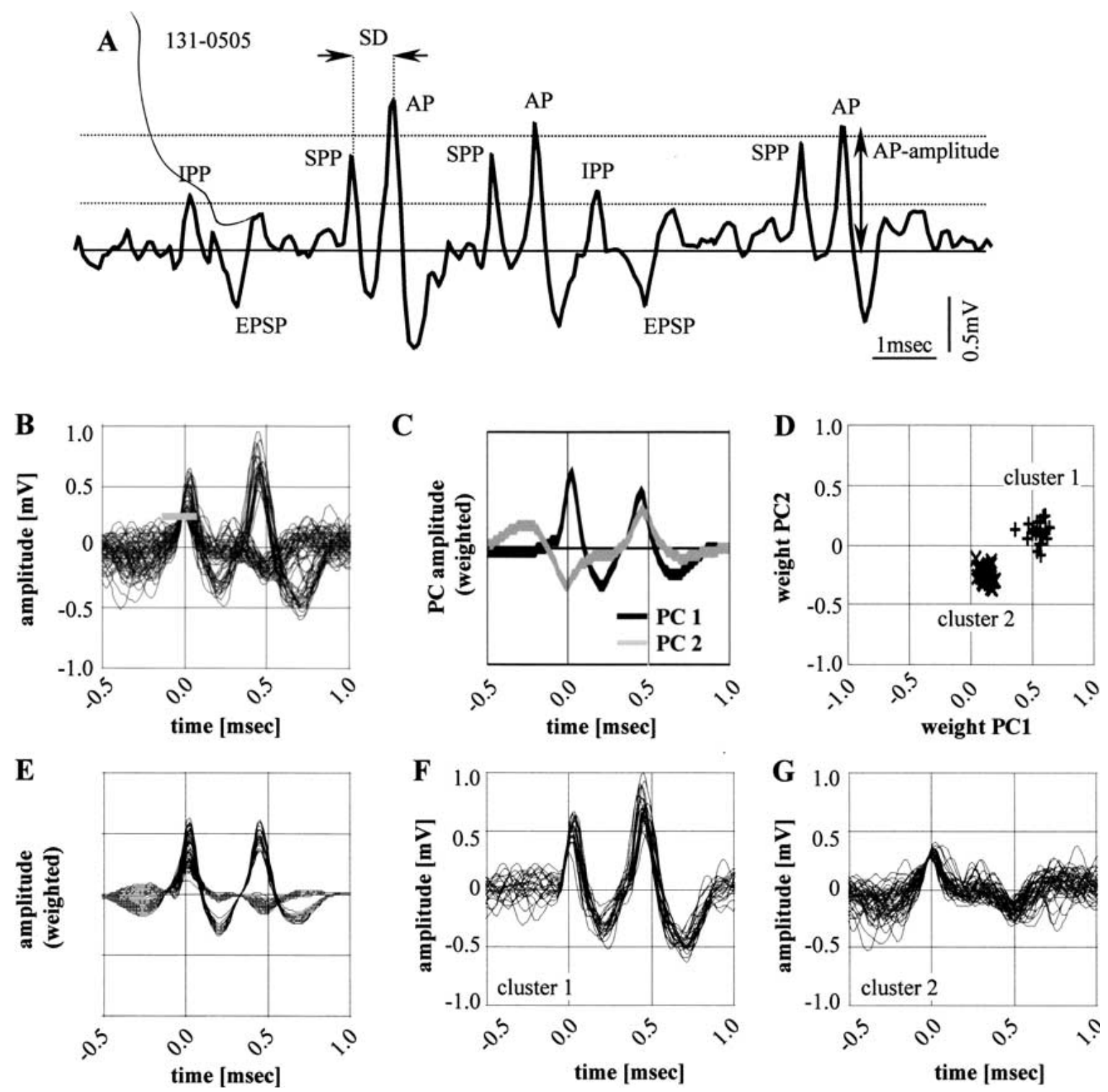

FIG. 4. Separation of pre- and postsynaptic signal components (unit 131-0505). A. Setting of trigger levels for the acquisition of response maps (like Fig. 1A). Presynaptic and postsynaptic discharge activity can be separated by different thresholds (lower and upper dotted lines for the pre- and postsynaptic activities, respectively). Additionally, a preset dead time of $1 \mathrm{~ms}$ prevents double triggers of postsynaptic action potentials following prepotentials in the prepotential recordings. SD: synaptic delay; AP: postsynaptic action potentials; SPP: spike-preceding prepotentials; IPP: isolated prepotentials; EPSPs: excitatory postsynaptic potentials. B-G. Separation of signal constituents using principal component analysis (PCA), same unit as in A, see Methods for further details. B. Superimposed waveforms of 202 discharges, aligned to the trigger time

Pre- and postsynaptic sound-evoked discharge rates were measured to $100-\mathrm{ms}$ pure tones presented $50 \mathrm{~dB}$ above threshold at the unit's CF (Fig. 5). Presynaptic discharge rates ranged from 48 to 868 pps (median: 355 [212, 486] pps), which was significantly larger than the respective postsynaptic rates (18 -334
(0.0). Trigger level was set to the onset slope of prepotentials, 0.25 $\mathrm{mV}$ (gray bar). Dead time between trigger events preset to $1 \mathrm{~ms}$. C. The first two principal components (PC1, PC2) of the signals shown in $\mathbf{B}$ multiplied by their root-mean-square weight. Together they give an accurate description of all recorded waveforms, here with an $87 \%$ efficiency. D. On the basis of PC1 and PC2 weights, the potentials are easily separated by a cluster algorithm into two clusters. E. Resynthesis of all 202 signals from the two PC time courses in $\mathbf{C}$ and the individual weight pairs in $\mathbf{D}$. The waveforms split up into two subsets, corresponding to IPP and preaction potential compounds. $\mathbf{F}$ and $\mathbf{G}$ show the original waveforms from $\mathbf{B}$ related to cluster 1 (preaction potential compounds) and to cluster 2 (IPPs), respectively.

pps; median: 194 [126, 230] pps; Wilcoxon signed rank test; $p \leq 0.001)$. For individual units, the failure rates ranged from $5 \%$ to $77 \%$ (median: $45[26,62] \%$ ). These were not reliably different from the failure rates for the spontaneous activity (Wilcoxon signed rank test; $p=0.322$ ). 


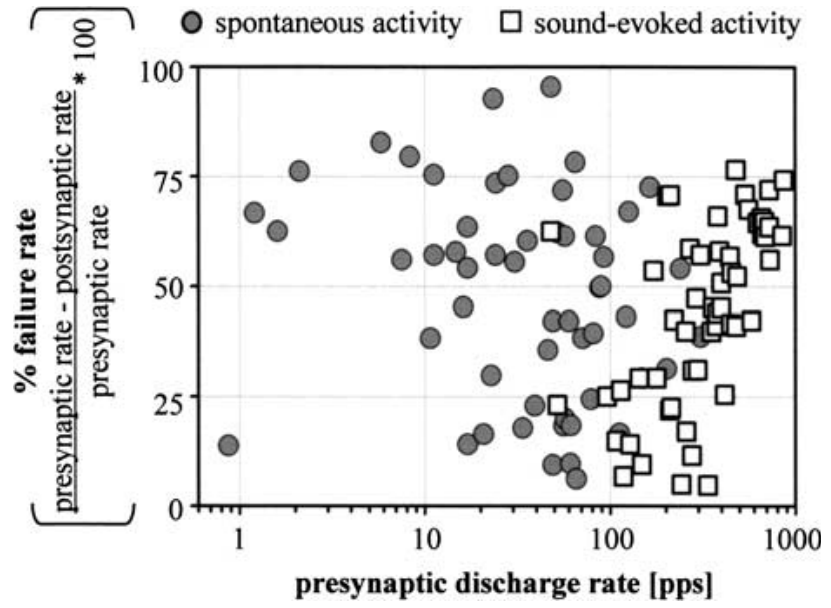

FIG. 5. Failures in spike transmission versus presynaptic spontaneous rates and sound-evoked rates for 54 units. Acoustic stimuli were set at the unit's CF and presented $50 \mathrm{~dB}$ above threshold.

\section{Characteristic frequency and tonotopy}

The distribution of the CFs in the gerbil's MNTB showed the same general mediolateral high-to-low frequency gradient described previously for rats (Sommer et al. 1993) and cats (Guinan et al. 1972b). Characteristic frequencies in the gerbil MNTB ranged from $1.0 \mathrm{kHz}$ in the lateral part of the nucleus to $54 \mathrm{kHz}$ in the medial part, with a relative overrepresentation in our sample of the frequency ranges between $1-5 \mathrm{kHz}$ (24\% of the units) and $25-30 \mathrm{kHz}$ (20\%). Ninety-four percent of the prepotential units $(51 / 54)$ had comparable pre- and postsynaptic CFs $(\Delta \mathrm{CF} \leq 0.25$ octave; $p=0.9)$. In only three units did the difference between pre- and postsynaptic CFs exceed 0.25 octave $(0.50,0.54$, and 0.63 octave).

\section{Tuning curves}

The tuning curves of MNTB units showed characteristic changes as a function of $\mathrm{CF}$, at both the presynaptic and postsynaptic levels (Fig. 6). Frequency selectivity was evaluated from the $Q$ values measured in $10-\mathrm{dB}$ steps from 10 to $60 \mathrm{~dB}$ above threshold $\left(Q_{\mathrm{x}}=\mathrm{CF} /\right.$ bandwidth $_{\mathrm{x}}$ ). Units with CFs between 1.0 and $6.0 \mathrm{kHz}$ $(n=15)$ had a mean threshold of $28 \pm 8 \mathrm{~dB}$ SPL and were mostly broadly tuned (medians: $Q_{10}=1.4, Q_{40}=$ 0.7 ; Fig. $6 \mathrm{~B})$. In the higher-frequency units $(\mathrm{CFs}=7-$ $54 \mathrm{kHz}), Q$ values varied as a function of threshold. One subset of units $(n=20)$ had a mean threshold of $20 \pm 5 \mathrm{~dB}$ SPL (Fig. 6D). These units showed fairly sharp frequency selectivity with median $Q_{10}$ and $Q_{40}$ values of 3.4 and 1.6, respectively. The other subset of high-frequency units $(n=19)$ had elevated thresholds $(49 \pm 11 \mathrm{~dB})$ and poorer frequency selectivity (medians: $Q_{10}=2.5, Q_{40}=1.3$, Fig. $6 \mathrm{~F}$ ). Units with these different tuning and threshold properties were frequently recorded in the same preparation, and the different types of high-frequency units could be encountered in the same penetration. The thresholds of the low-CF units and the broadly tuned high-CF units were elevated by approximately $30 \mathrm{~dB}$ above the respective values of the gerbil's behavioral audiogram (Ryan 1976), whereas the narrowly tuned high-CF units showed a mean threshold elevation of about $15 \mathrm{~dB}$.

When the tuning characteristics of the presynaptic activity were measured (SPPs and IPPs), units showed almost the identical grouping as the postsynaptic activities (Fig. 6A, C, E). However, a comparison of the pre- and postsynaptic $Q$ values for individual units showed a pre- to postsynaptic increase in frequency selectivity (Fig. 7). Frequency selectivity was significantly sharper postsynaptically than presynaptically at all stimulus levels $\geq 20 \mathrm{~dB}$ above threshold (Wilcoxon signed rank test: all $p$ 's $\leq 0.05$ )

\section{Rate-level functions}

The encoding of sound intensity by discharge rate is reflected in a neuron's rate-level function (RLF). In the present study, pre- and postsynaptic RLFs were calculated by measuring discharge rates to 15 sound pressure levels (generally between 20 and $90 \mathrm{~dB}$ SPL) presented five times each at the unit's CF. The RLFs could be classified as either monotonic or nonmonotonic (Fig. 8). A RLF was defined as being nonmonotonic if the discharge rate averaged across the three highest intensities was less than $80 \%$ of the unit's maximal discharge rate (Fig. 8D). Monotonic RLFs were divided into two subgroups, "plateau" RLFs, in which the discharge rate increased up to a given stimulus level but showed no further increase at higher intensities (Fig. 8A, C) and "steadily increasing' RLFs, in which discharge rates increased steadily up to $90 \mathrm{~dB}$, the highest intensity tested (Fig. 8B). No correlation was observed between the unit's CF and the type of RLF.

Figure 9 shows the frequency of occurrence of the different types of RLFs. With respect to their presynaptic activity, $56 \%(30 / 54)$ of the prepotential units showed monotonic rate-level functions with plateaus (Fig. 8A), and 20 units (37\%) showed steadily increasing RLFs (Fig. 8B). Only 7\% (4/54) had nonmonotonic RLFs. With regard to their postsynaptic activity, $70 \%(38 / 54)$ of the units had plateau-type monotonic RLFs (Fig. 8C) and only 6\% (3/54) showed steadily increasing RLFs. Twenty-four percent $(13 / 54)$ were categorized as nonmonotonic (Fig. $8 \mathrm{D})$. The discharge rates of these nonmonotonic RLFs reached their maximum at a certain intensity and decreased toward higher intensities by about 26\%. In none of the nonmonotonic units did the reduced levels of activity at higher intensities ever fall below the respective spontaneous rates. 


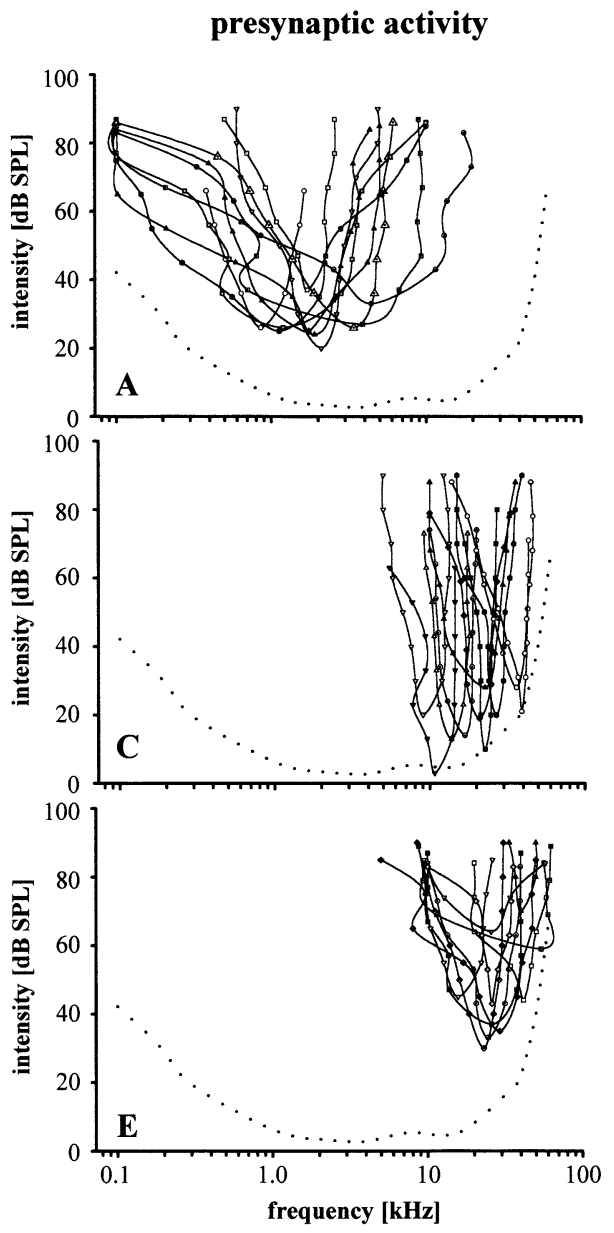

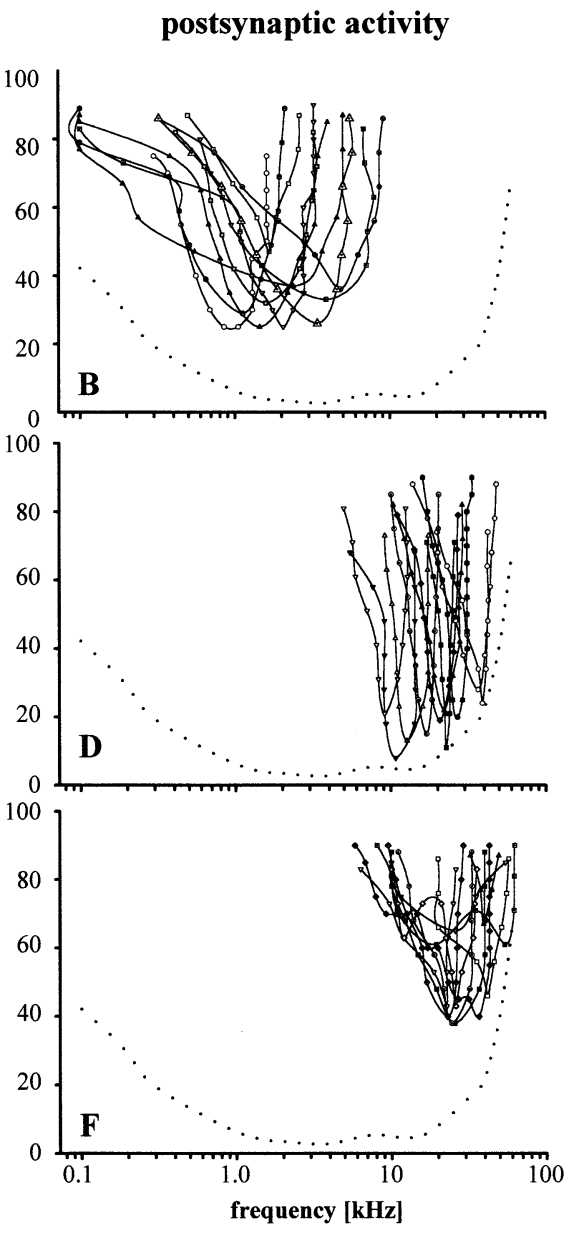

FIG. 6. Examples of different presynaptic (A, C, E) and their corresponding postsynaptic (B, D, F) frequency tuning curves (10 each). Dotted lines show the behavioral audiogram of the gerbil (Ryan 1976). A, B. Broadly tuned (often) symmetric tuning curves are dominant between 1 and $6 \mathrm{kHz}$. C, D. One portion of highfrequency units ( $\mathrm{CF}=14-39 \mathrm{kHz})$ is characterized by narrow tuning and low thresholds. E, F. Other high-CF units $(\mathrm{CF}=18-53 \mathrm{kHz})$ again have broader tuning and elevated thresholds. Pre- and postsynaptic tuning curves of the same unit are marked by the same symbols.
Direct comparison of the pre- and postsynaptic RLFs for individual neurons showed that the respective RLFs differed from each other in half of the units examined (Fig. 9). Most notably, $42 \%(n=16 / 38)$ of MNTB neurons with plateau monotonic RLFs received their excitatory input from afferents with steadily increasing RLFs. About 70\% $(n=9 / 13)$ of the MNTB neurons with nonmonotonic RLFs were associated with afferents having plateau RLFs.

\section{Response pattern}

In response to tone burst stimulation at the unit's CF and at a sound pressure level of $80 \mathrm{~dB}$ SPL, all units showed phasic-tonic response patterns in both their pre- and postsynaptic activity (Fig. $10 \mathrm{~A}-\mathrm{F}$ ). Displaying these responses using small bin widths (Fig. $10 \mathrm{C}$, $\mathrm{F}$ insets) indicates that these responses had the same primarylike notch patterns that have previously been described for MNTB units and globular bushy cells in the VCN (Smith et al. 1991, 1998; Tsuchitani 1997). The pre- and postsynaptic responses of each unit showed a reliable initial action potential with a constant latency. However, postsynaptically the discharge rate following the initial spike was greatly decreased (Fig. 10 A, B and D, E). As can be seen from the PSTHs, this is reflected as an enhancement in the magnitude of initial-spike response relative to the remainder of the sound-evoked activity (Fig. $10 \mathrm{C}, \mathrm{F}$ ). To quantify this, the number of spikes in the first 1ms bin was expressed as a percent of the total number of spikes in the first $100 \mathrm{~ms}$ of the response. This peak-over-total measure was significantly larger postsynaptically (median: $1.8 \%[1.4,3.8]$ ) than presynaptically (median: $0.9 \%[0.8,1.6])(n=54$; Wilcoxon signed rank test; $p \leq 0.001$ ).

The IPPs themselves typically showed sustained responses that lacked any prominent phasic component. Additionally, the latency of the initial discharge was more variable (Fig. $10 \mathrm{H}, \mathrm{J}$ ). Thus, the IPPs did not exhibit the primarylike pattern notch pattern that would be expected if these signals reflected the discharge of a separate calyceal fiber or MNTB unit.

\section{Phase-locking fidelity}

It is generally reported that sound frequencies below $5 \mathrm{kHz}$ are encoded in the synchronization of dis- 


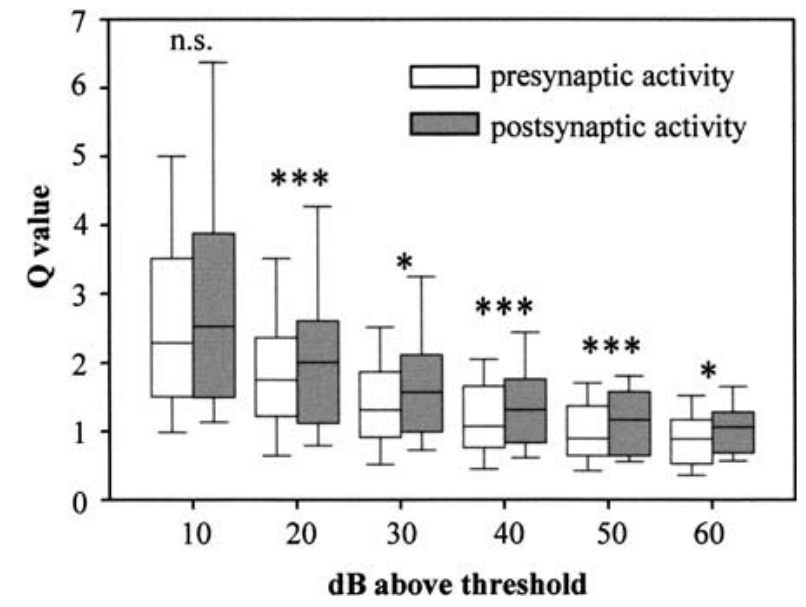

FIG. 7. Decrease in frequency tuning ( $Q$ values) as a function of sound pressure level. Data are shown as boxplots with the median (horizontal lines), $10 \%, 25 \%, 75 \%$, and $90 \%$ percentiles. $Q$ values were measured in 10-dB steps above threshold, showing a steady decrease with increasing levels $(n=54)$; at higher levels, $n$ is reduced because the test level was restricted to below $90 \mathrm{~dB}$ SPL (minimal: $n=29$ at $60 \mathrm{~dB}$ above threshold). For $Q_{20}$ and higher, the postsynaptic values significantly exceed the presynaptic ones, indicating a postsynaptic increase in frequency selectivity (Wilcoxon signed rank test, $* * *: p \leq 0.001 ; *: p \leq 0.05)$.

charges to the period of the acoustic stimulus (Goldberg and Brown 1969). The majority of MNTB units in our study had CFs above $5 \mathrm{kHz}$. The small number of low-frequency MNTB neurons from which we recorded ( $\mathrm{CF}=1-4 \mathrm{kHz}, n=12)$ showed phaselocked responses to low-frequency stimuli. Additionally, units with somewhat higher CFs $(4 \mathrm{kHz} \leq \mathrm{CF} \leq$ $6.5 \mathrm{kHz}, n=3$ ) showed strong phase-locking in the region of their low-frequency tails. Figure 11 shows the discharges of a prepotential unit to repetitive acoustic stimulation with a sinusoid tone $(100 \mathrm{~Hz})$. Presynaptic discharges were found to be synchronized with each period of the stimulus, but occasionally solely an IPP occurred instead of a pre\&action potential compound.

To quantify the degree of phase-locking, the synchronization index (SI) was computed for the lowand medium-frequency MNTB units (Goldberg and Brown 1969; Knipschild et al. 1992). Figure 12A, B shows an example of a low-frequency MNTB unit $(\mathrm{CF}=3.8 \mathrm{kHz})$ with consistent phase-locking in its low-frequency tail up to $1.7 \mathrm{kHz}$. At frequencies below $1.2 \mathrm{kHz}$, the degree of phase-locking was higher postsynaptically than presynaptically. This is exemplified by the period histograms shown for respective pre- and postsynaptic frequency/intensity combinations (Fig. 12A, B insets). In addition, significant phase- locking extended to lower intensity levels postsynaptically than presynaptically, even occurring at some intensities below the frequency-threshold curve (dashed line in Fig. 12B).
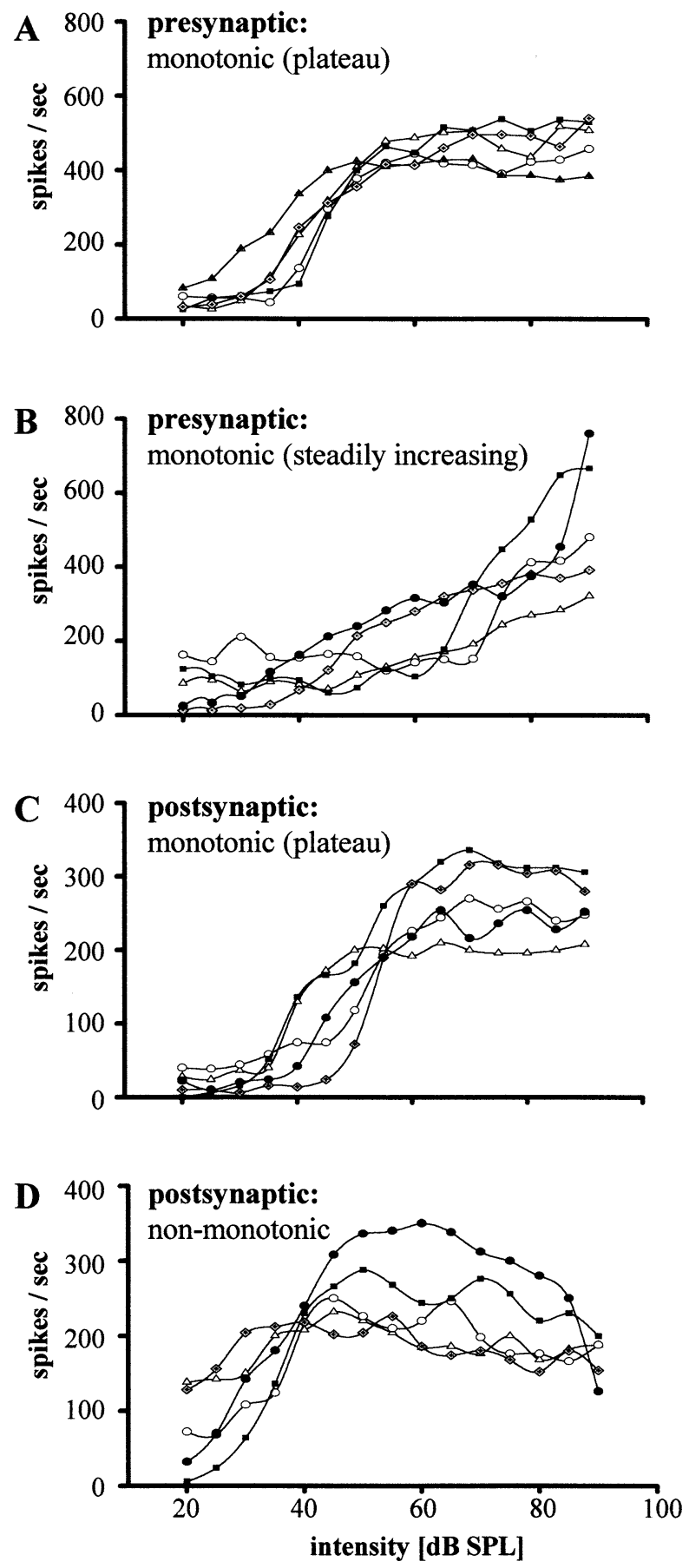

FIG. 8. Representative rate-level functions (RLFs) of presynaptic and postsynaptic recordings. A. Monotonic (plateau) presynaptic RLFs B. Monotonic (steadily increasing) presynaptic RLFs. C. Monotonic (plateau) postsynaptic RLFs. D. Nonmonotonic postsynaptic RLFs. Note the different scales in A and B vs. C and D.

To quantitatively compare the differences in phase-locking between pre- and postsynaptic activities, the mean SI was computed for each of seven different frequency ranges. The results of this analysis for ten low-to-midfrequency units $\quad(\mathrm{CFs}=1.0-6.5$ $\mathrm{kHz}$ ) showed that phase-locking was significantly greater postsynaptically than presynaptically for fre- 


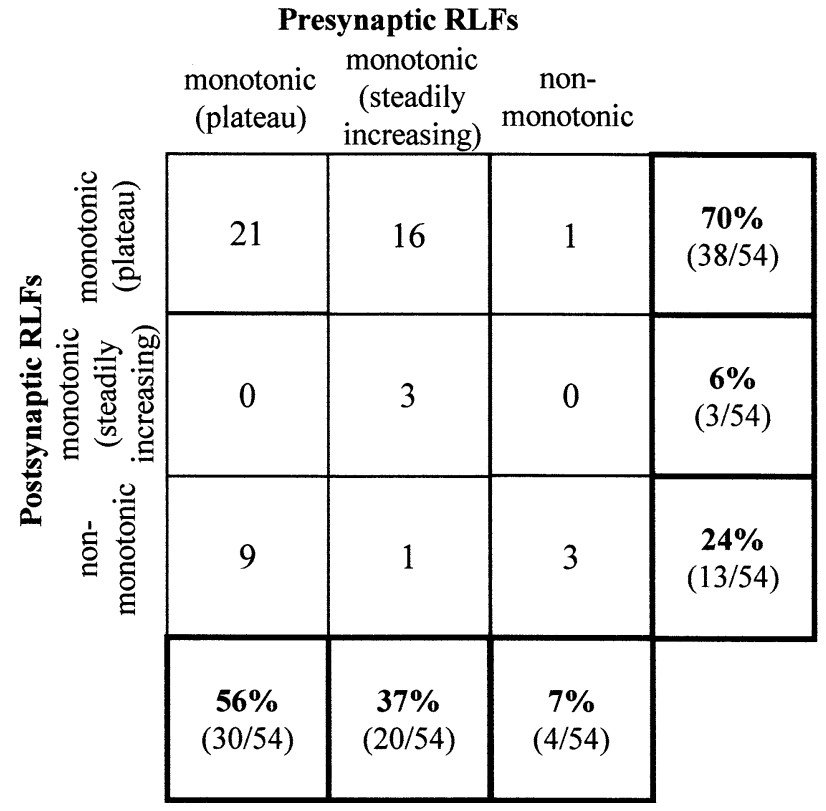

FIG. 9. Overall distribution of RLF types and their pre- and postsynaptic combinations.

quencies below $0.8 \mathrm{kHz}$ (Mann-Whitney rank sum test, all $p$ 's $\leq 0.001$; Fig. 12C). Toward higher frequencies, the pre- and the postsynaptic SI values converged and were not reliably different.

In summary, the present results demonstrate that pre- and postsynaptic activities differed in a number of different ways: (a) postsynaptic spontaneous and sound-evoked activity is reduced, (b) postsynaptic frequency selectivity is increased and postsynaptic phase-locking is improved, and (c) the phasic component of the postsynaptic response is enhanced. These differences between pre- and postsynaptic activities indicate that synaptic transmission at the CN-MNTB synapse does not occur in a one-to-one fashion.

\section{RESPONSES TO TWO-TONE STIMULATION}

One factor that could contribute to the differences between pre- and postsynaptic activity is neuronal inhibition. In extracellular recordings, inhibitory influences can be shown only for discharging neurons, i.e., as a reduction in a neuron's spontaneous or sound-evoked activity. For the following series of experiments, the two-tone stimulus paradigm (introduced by Arthur et al. 1971) illustrated in Figure 13 was used to demonstrate the occurrence of acoustically evoked inhibition within the MNTB. A probe-tone (100 ms) was set at the unit's CF and presented at 20 $\mathrm{dB}$ above threshold. This stimulus produced an increased level of discharge that allowed inhibitory effects to be detected (Fig. 13A, B). A second tone burst, the test-tone, was then added to the probe-tone, and its effect on the probe-tone-evoked activity was measured (Fig. 13C, D). Test-tones were typically presented outside the excitatory response area at intensities 50-70 dB above threshold. The test-tone was $40 \mathrm{~ms}$ long and delayed by $30 \mathrm{~ms}$ relative to the onset of the probe-tone, i.e., the test-tone was embedded in the excitatory stimulus during the tonic component of the unit's response. This allowed both the amount of test-tone-induced reduction in activity and the time course of the reduction in activity to be measured. Test-tone-induced reduction of MNTB neuronal firing can occur because of a decreased presynaptic activity (due, for example, to cochlear two-tone suppression or two-tone inhibition in the $\mathrm{CN}$ ) or inhibition of MNTB cells or both processes.

Presynaptically, the response can be determined by both the effects of cochlear two-tone suppression and of two-tone inhibition at the level of the CN. Inhibition of MNTB neuronal firing is indicated when the test-tone-induced reduction of the probe-tone-evoked response is greater postsynaptically than presynaptically (e.g., see Fig. 17).

Figure 14 provides one clear example of a unit in which presentation of the test-tone caused a large reduction in action potential firing without affecting the level of presynaptic activity. All graphs in Figure 14 are based upon the same recordings, acquired with the frequency of the probe-tone set at the unit's CF $(27 \mathrm{kHz}, 65 \mathrm{~dB})$ and the test-tone set at a frequency/ intensity combination that was outside the unit's preand postsynaptic excitatory response areas $(30 \mathrm{kHz}$, $65 \mathrm{~dB})$. The test-tone caused a large reduction in the postsynaptic probe-tone-induced activity (Fig. 14A, B) but had little or no effect on the level of presynaptic discharges (Fig. 14C, D).

In order to quantify the differential effect of the test-tone on presynaptic activity and postsynaptic activity of 54 prepotential units, the failures of postsynaptic spike generation were compared under three different conditions: no stimulation (spontaneous activity), probe-tone stimulation (at CF), and two-tone stimulation (test-tone presented outside the unit's pre- and postsynaptic excitatory response areas) (Fig. 15). As noted previously in the section on spontaneous activity, the median failure rate during spontaneous discharges was 52\% [29; 64]. Following probe-tone stimulation, pre- and postsynaptic activities increased but the failure rates were not significantly changed (median: 45\% [26; 62]). Addition of the test-tone produced a large reduction in postsynaptic discharges and a significant increase in failure rates (median: 74\% [60,77]). The large increase of failure rates during two-tone stimulation provides evidence for an acoustically evoked inhibition of MNTB neuronal firing. 


\section{presynaptic activity (SPPs \& IPPs)}

A

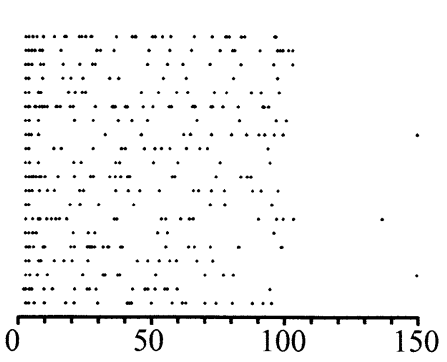

B

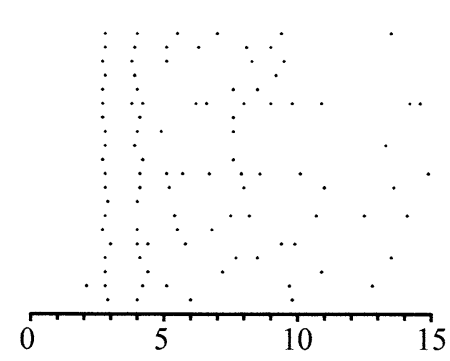

C

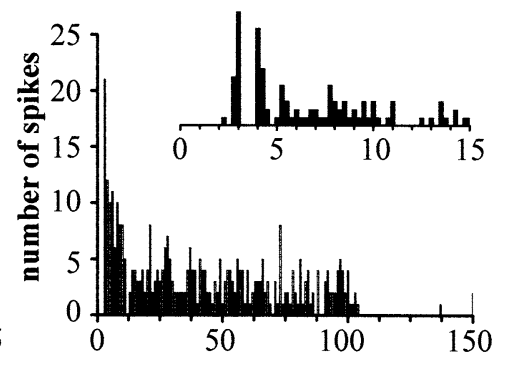

\section{postsynaptic activity (action potentials)}

D

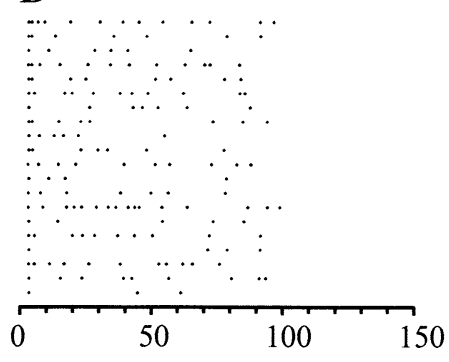

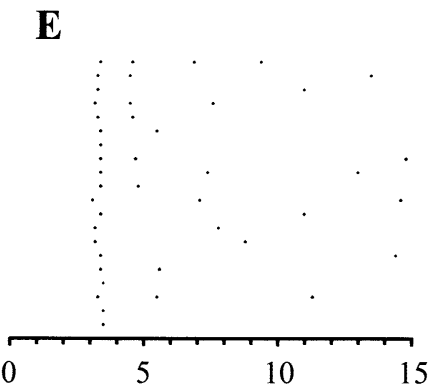

\section{IPPs}

\section{H}

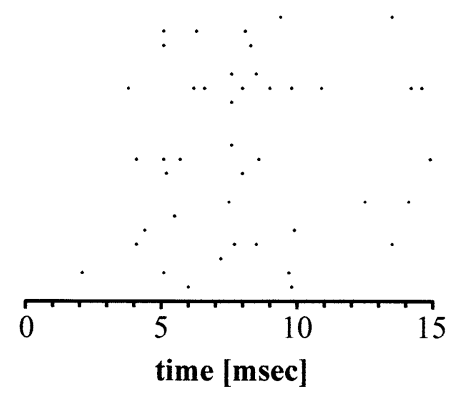

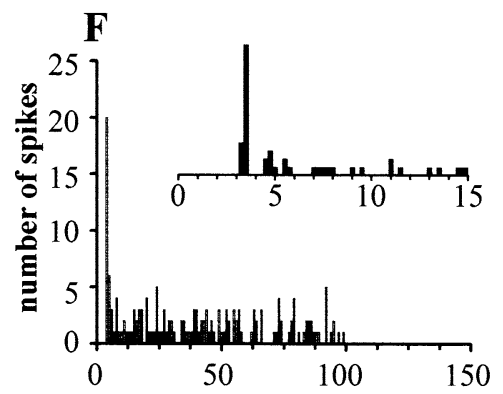

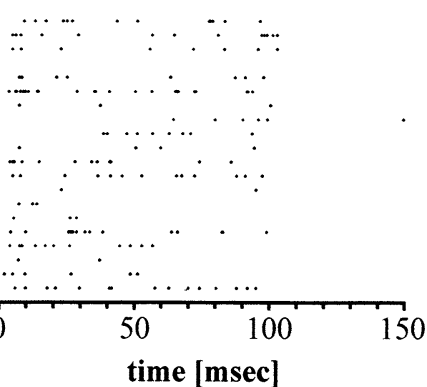

FIG. 10. Dot raster displays and PSTHs of unit G134-18 at CF $=9.0$ $\mathrm{kHz}, 50 \mathrm{~dB}$ SPL, $100 \mathrm{~ms}$ tone burst, 20 stimulus repetitions. The left column shows dot raster displays of which the respective first $15 \mathrm{~ms}$ are given in the middle column with an enlarged time scale. Right column PSTHs (bin width $=1 \mathrm{~ms}$ ). The insets show the first $15 \mathrm{~ms}$ of

\section{IPPS}

The behavior of IPPs under two-tone stimulation provides additional evidence that IPPs and SPPs are the same signal and not discharges of different neural elements. As illustrated in Figure 14E, F, presentation of the test-tone during probe-tone stimulation was associated with an increase in the number of IPPs. The increased number of IPPs corresponded tem-

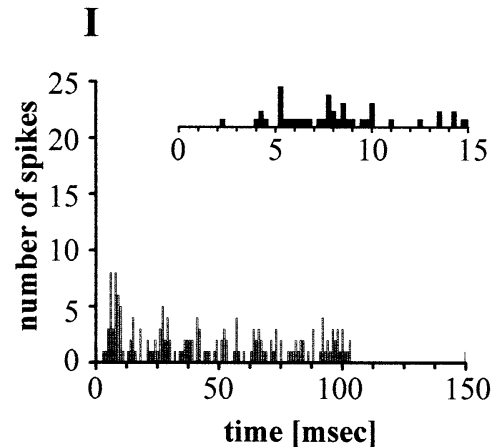

the respective PST histograms with a bin width of $0.25 \mathrm{~ms}$. A-C. Presynaptic recordings (SPP and IPP). D-F. Postsynaptic recordings. G-I. IPPs. Note that the onset component is shortened postsynaptically due to failures of spikes following the first spike. The IPP activity lacks a sharp onset response.

porally with a reduction in the number of SPPs, which is similar to the reduction of postsynaptic action potentials (Fig. 14A, B). One possible explanation of this correlated change in the number of SPPs and IPPs is that both arise from different neural elements that were oppositely affected by the test-tone. That this is not the case is indicated by the fact that the test-tone was outside the excitatory response areas of the proposed neural elements generating the pre- 
A $134-0210$

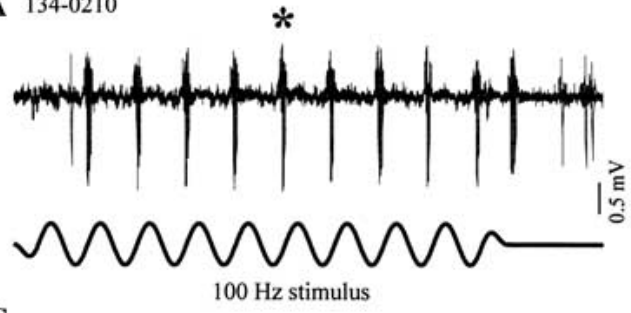

B

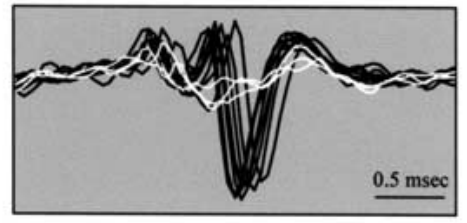

C
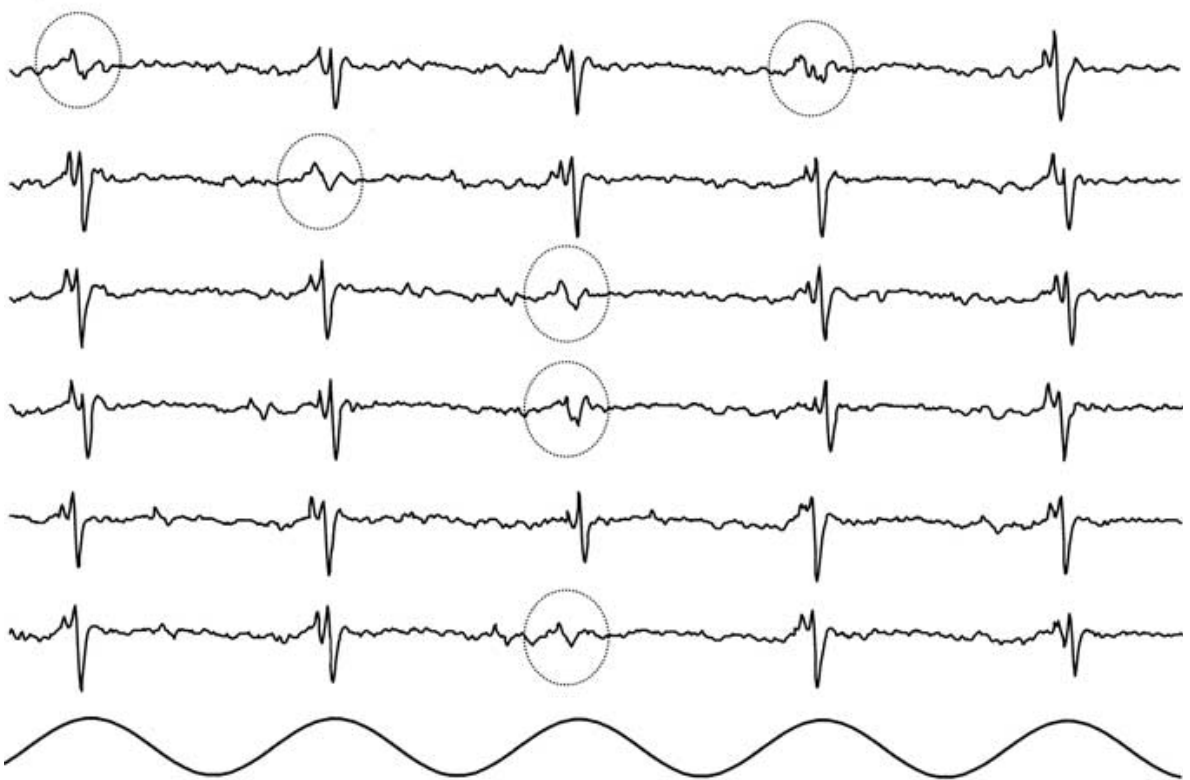

$5.0 \mathrm{msec}$

FIG. 11. Discharges of a midfrequency MNTB unit (G134-0210; CF $=6.5 \mathrm{kHz})$ which shows phase-locked responses to low-frequency sinusoids (here $100 \mathrm{~Hz}$ ). A. Twenty superimposed spike trains. Signals marked by an asterisk are shown with an enlarged time scale in $\mathbf{B}$, in which IPPs are shown by white lines and preaction potential compounds by black lines. C. Each signal period caused a discharge, mostly preaction potential compounds but occasionally only IPPs (identified by circles). synaptic activity and the postsynaptic activity. That is, presentation of the test-tone alone had no affect on IPPs (Fig. 14G, H). The more likely explanation is that SPPs and IPPs are prepotentials of the same presynaptic terminal. Because the test-tone produced a reduction in postsynaptic firing, the number of prepotentials classified as SPPs (i.e., those prepotentials immediately followed by an action potential) and IPPs (prepotentials not followed by a spike) would decrease and increase, respectively.

\section{Time course of discharge reduction}

It is generally observed that the time between the presentation of an excitatory acoustic stimulus and the onset of the evoked neural response decreases as a function of increasing sound intensity (e.g., Rhode and Smith 1986). If acoustically evoked inhibition contributes to the reduction in postsynaptic discharge rate, one would expect that the latency of the inhibitory effect would also decrease with increasing sound intensity. To examine this, the inhibitory and excitatory latencies were measured in 10 units. For this purpose, the excitatory latency was defined as the time between the onset of the probe-tone and the time at which the neuron reached its half-maximal discharge rate. The inhibitory latency was defined as the time between the onset of the test-tone and the time at which the tonic component of the neuron's response was reduced to its half-maximal level (Fig. 16A). Because test-tone-induced reduction of discharge rates occurred mainly at test-tone levels greater than $50 \mathrm{~dB}$ (see following section on inhibitory sidebands), the inhibitory latency was measured only at higher intensity ranges.

Excitatory and inhibitory latencies both decreased with increasing sound intensity (Fig. 16B, C). Large reductions in the excitatory latency occurred for probe-tone intensities up to 30-40 dB SPL, whereas at higher intensities the latencies remained relatively constant ( $>50$ dB SPL; median: $3.4[3.1,3.6] \mathrm{ms}$; $n=10$ ). Inhibitory latencies similarly decreased with increasing test-tone levels between 50 and $90 \mathrm{~dB}$ SPL. Generally, inhibitory latencies were much more variable between units than were excitatory latencies. On average, inhibitory latencies were significantly longer than excitatory latencies ( $>50 \mathrm{~dB}$ SPL; median: 6.1 $[4.9,8.7] \mathrm{ms} ; n=10$; Wilcoxon signed rank test $p=0.002$; Fig. 16C).

\section{Inhibitory sidebands}

In order to examine how MNTB neurons integrate their excitatory and inhibitory inputs across fre- 

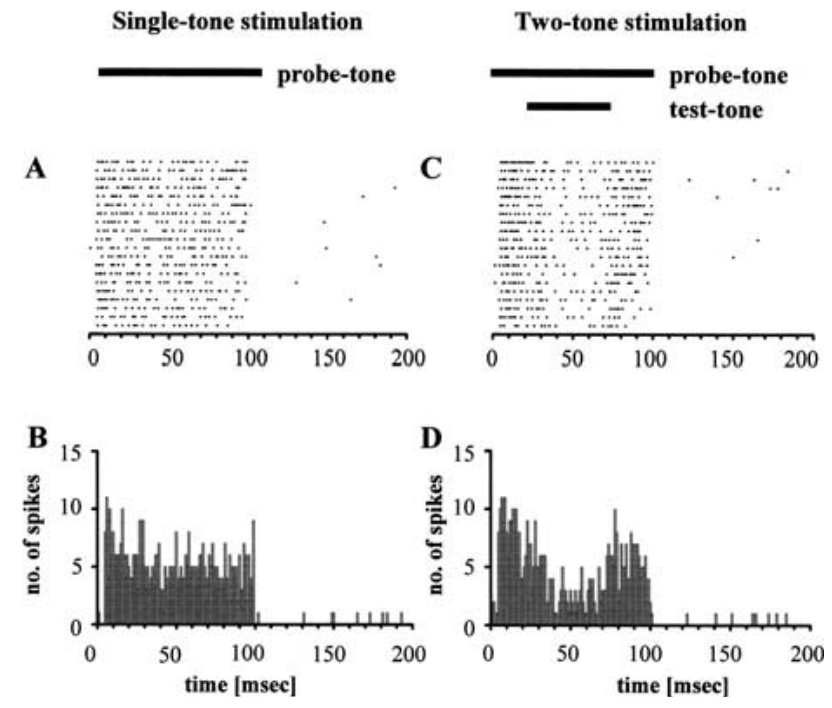

FIG. 13. Two-tone stimulus paradigm, postsynaptic recording of unit 131-0908/14. A, B. Single-tone stimulation. C, D. Two-tone stimulation. Probe-tones (100 ms, indicated by black bars) were presented $20 \times$ at the unit's CF $(3.4 \mathrm{kHz}) 20 \mathrm{~dB}$ above threshold. Testtones $(40 \mathrm{~ms}$, indicated by short black bar for C and D, $7.0 \mathrm{kHz}, 80$ $\mathrm{dB}$ SPL) were added to the probe-tone with a delay of $30 \mathrm{~ms}$. The dot raster display and the PSTH (bin width $=1 \mathrm{~ms}$ ) show reduced spike rates during the presentation of the test-tone.

level of the MNTB and, thus, are reflected in its afferent input, to be distinguished from inhibition that is expressed in the MNTB itself.

The results from one representative unit are shown in Figure 17. As can be seen from the two top panels, the unit showed greater frequency selectivity in its postsynaptic than in its presynaptic excitatory response areas (Fig. 17A, B). For the two-tone stimulation, the presynaptic (left column) and the postsynaptic activities (right column) are shown sideby-side for comparable probe-tone levels between 20 and $50 \mathrm{~dB}$ above threshold (Fig. 17C-J). Test-toneinduced reductions of discharge activity formed inhibitory sidebands below and/or above the CF in both presynaptic and postsynaptic recordings. A comparison of the respective sidebands clearly shows that at all probe-tone levels the inhibitory sidebands were more extensive, i.e., covered a wider frequency range, postsynaptically than presynaptically. Additionally, the overall area of the inhibitory sidebands increased with increasing probe-tone levels.

In $70 \%(38 / 54)$ of the units examined, the postsynaptic inhibitory sidebands were more extensive than the presynaptic ones. In all these neurons, these sidebands flanked or overlapped the excitatory response area at its high- and/or the low-frequency sides. In many neurons, the postsynaptic sideband thresholds decreased with increasing probe-tone levels. Additionally, in these neurons the area of the inhibitory sidebands steadily increased with increasing probe-tone levels (e.g., Fig. 17). Other units showed the strongest reduction and the lowest inhibitory thresholds at medium probe-tone levels (20$30 \mathrm{~dB}$ above threshold). These observations indicate that the dependence of the inhibitory influence on probe-tone level varied between units. The remaining $30 \%$ of the units $(16 / 54)$ did not show significant differences between pre- and postsynaptic inhibitory sidebands.

\section{DISCUSSION}

The present results showed that the pre- and postsynaptic discharge activity of MNTB neurons differed from each other in a number of respects. Spontaneous and sound-evoked discharge rates were greater presynaptically than postsynaptically, and frequency tuning was sharper postsynaptically. Furthermore, noticeable differences were found between the respective temporal response patterns, e.g., postsynaptically the initial part of the phasic response component was relatively enhanced and pre- and postsynaptic RLFs differed significantly from each other in about half of the neurons examined. The fidelity of phase-locking to sound frequencies up to 1 $\mathrm{kHz}$ showed a pre- to postsynaptic improvement. These differences between pre- and postsynaptic response characteristics indicate that transmission at the CN-MNTB synapse does not occur in a one-toone manner. This is consistent with the idea that the discharge activity of MNTB neurons is the result of the integration of their excitatory and inhibitory inputs. Overall, these results indicate that the MNTB constitutes an additional modulation site in the auditory pathway where the strength and the time course of the contralateral inhibitory input to the LSO are adjusted.

\section{Complex waveforms in MNTB unit discharges}

All conclusions drawn in this study were based on the differential analysis of the activity of MNTB neurons and of their excitatory afferent terminals, the calyces of Held. The differential analysis was possible because the extracellularly recorded signals were composed of two distinct components reflecting the respective preand postsynaptic discharge activities. This distinction was first reported by Pfeiffer (1966) in recordings from AVCN neurons, which also receive their main excitatory afferent input from large-sized endbulb terminals. Pfeiffer also coined the term "prepotential" for the presynaptic component of the signal complexes. Guinan and $\mathrm{Li}$ (1990) subsequently confirmed the presynaptic nature of the prepotentials. By using retrograde electrical stimulation while recording from the MNTB of cats, they were able to selectively evoke 
Postsynaptic activity (Action Potentials)

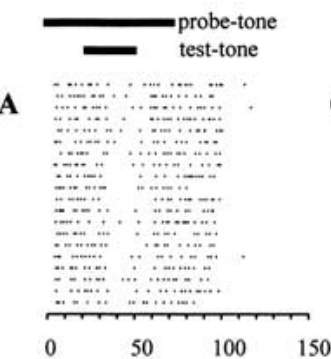

Presynaptic activity (SPPs \& IPPs)

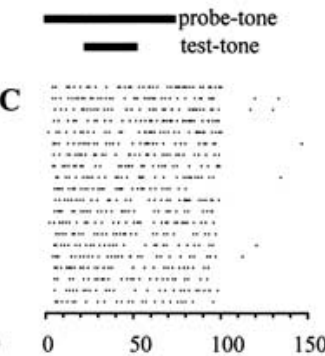

IPPs

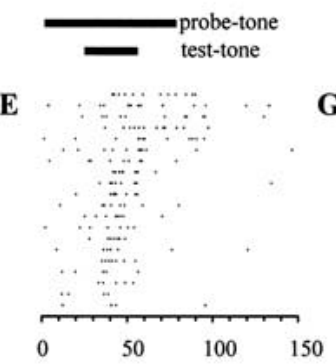

IPPs

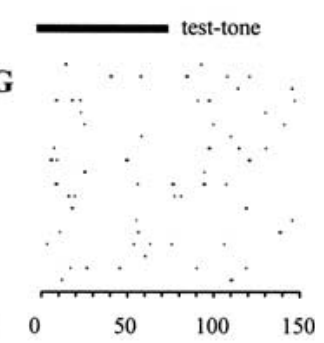

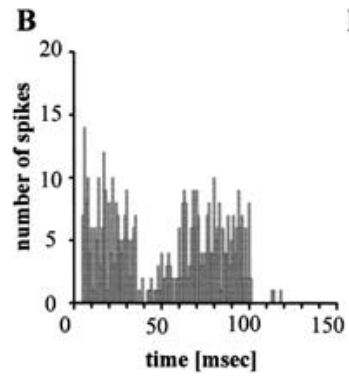
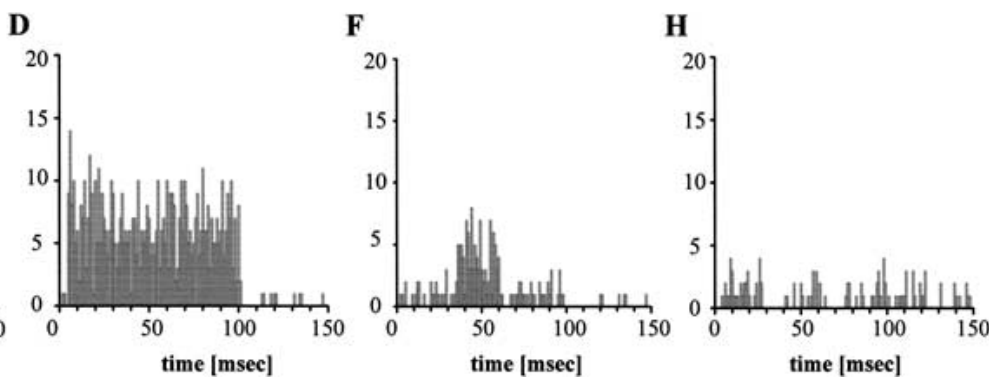

FIG. 14. Single-unit recording (84-0316; CF = 27 kHz) under twotone stimulation. Dot raster (top, 20 stimulus runs) and respective PSTH (bottom, bin width = $1 \mathrm{~ms}$ ). Probe-tone: $27 \mathrm{kHz}$ at $65 \mathrm{~dB}$ SPL, $100 \mathrm{~ms}$; test-tone: $30 \mathrm{kHz}$ at $65 \mathrm{~dB}$ SPL (outside the excitatory response area), duration $40 \mathrm{~ms}$ delayed by $30 \mathrm{~ms}$. A, B. Postsynaptic activity (APs). C, D. Presynaptic activity (SPPs and IPPs). E, F. Isolated

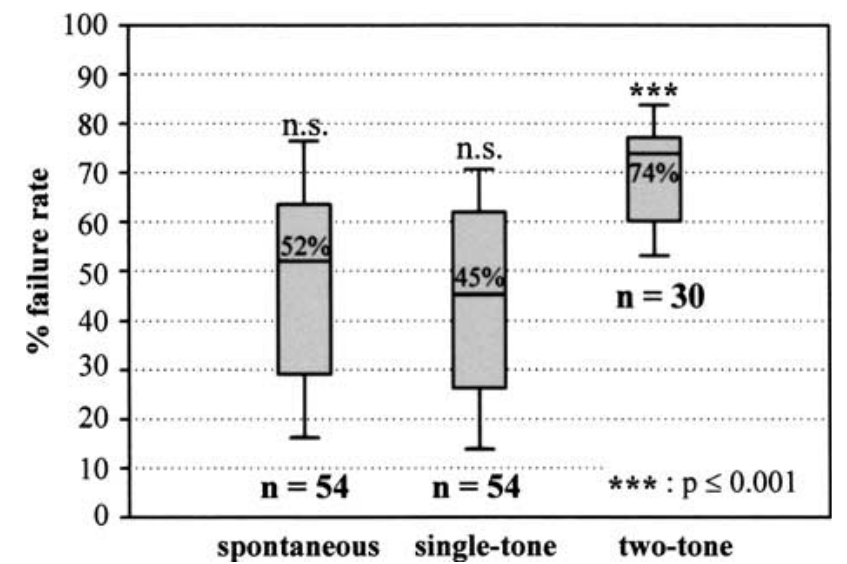

FIG. 15. Distribution of failure rates of 54 MNTB units acquired under three different stimulus conditions: no stimulus (spontaneous activity), single-tone stimulation (probe-tone at CF, $50 \mathrm{~dB}$ above threshold), and two-tone stimulation (probe-tone and test-tone). Failure rates differ only slightly between spontaneous- and probetone-evoked activity but increase under two-tone stimulation. Data are shown as boxplots with the median (horizontal lines), 10\%, 25\%, $75 \%$, and $90 \%$ percentiles.

only the postsynaptic component of the signal complexes (Guinan and Li 1990). Wu and Kelly (1992) subsequently succeeded in showing that the prepotential reflected the discharge activity of the presynaptic calyx. Their study was based on orthodromic electrical prepotentials (IPPs). Note that the test-tone has no noticeable effect on the presynaptic discharge activity but significantly reduces the postsynaptic discharge activity and at the same time causes an accumulation of IPPs. G, H. The test-tone, when presented alone (100 ms duration), does not affect the IPP activity.

stimulation and extracellular recordings from MNTB neurons in mouse brain slices. By preventing synaptic transmission, they were able to selectively suppress the postsynaptic response while maintaining the presynaptic component of the signal complex.

The waveforms of the extracellularly recorded pre\&action potential compounds described in these previous studies differed somewhat from each other, but these differences most probably only reflect different recording conditions (Pfeiffer 1966; Guinan and Li 1990; Wu and Kelly 1992). The form and the polarity of extracellularly recorded signals depend on the input impedance of the recording electrode and also on the spatial relation between the tip of the electrode and the recorded neuron. Signals with a positive presynaptic component followed by a solely negative postsynaptic component were described by Guinan and Li (1990). In the present study, similar signals were observed when using electrodes with impedances of approximately $6 \mathrm{M} \Omega$. An increase in electrode impedance resulted in the signal waveforms becoming more biphasic, similar to the waveforms shown by Wu and Kelly (1992).

Prepotential units indicating both the pre- and postsynaptic neuronal discharge activity are not unique to electrophysiological studies of the central auditory system. Such units have also been described in the visual system, e.g., in the lateral geniculate 
A
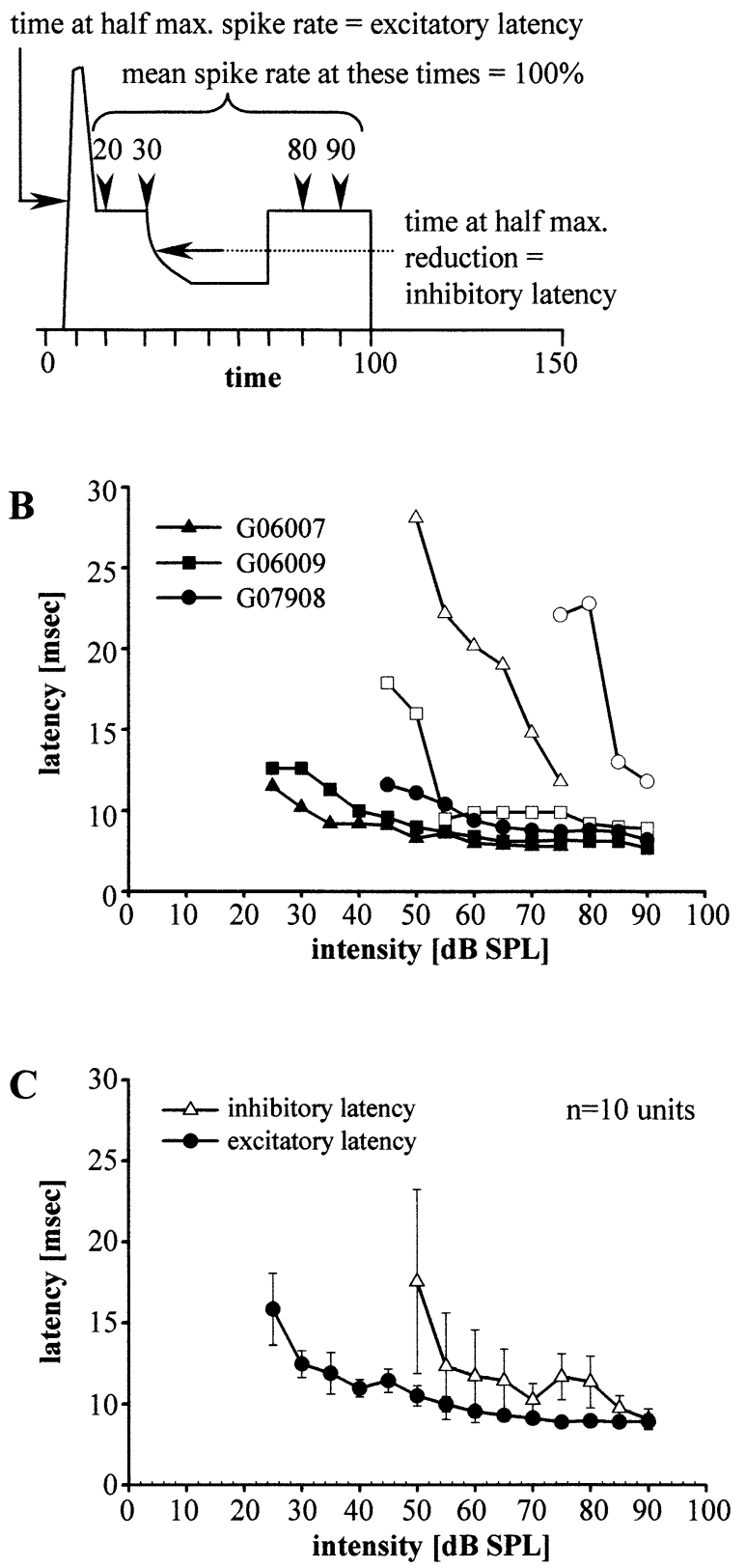

FIG. 16. Excitatory and inhibitory latencies. A. Evaluation method: Time of half-maximal rate increase was taken as the excitatory latency, and the time of the half-maximal activity reduction during the tonic component defined as the inhibitory latency. For the latter, the mean spike rate at 20,30,80, and 90 ms was taken as reference. B. Level-dependent variations of excitatory (closed symbols) and inhibitory (open symbols) latencies shown for three units. For excitatory latencies, the abscissa refers to probe-tone intensity, for the inhibitory, to test-tone intensity. Inhibitory latencies were acquired with probe-tone levels of $35 \mathrm{~dB}$ SPL (G06007 and G06009) and 55 dB SPL (G07908). C. Mean excitatory and inhibitory latencies for ten units (error bars $=$ SEM).

nucleus of the cat (Bishop et al. 1958; Hubel and Wiesel 1961; Cleland et al. 1971) and macaque monkey (Lee et al. 1983), and in the somatosensory system of the rat (Vahle-Hinz et al. 1994).
Basis for assuming the isolated prepotentials are prepotentials

In contrast to earlier electrophysiological studies in the MNTB that also documented prepotential units, the present study reports a second type of signal that was frequently recorded together with the pre\&action potential compounds. These signals were similar in their shape to the spike-preceding prepotentials. However, they were not immediately followed by postsynaptic action potentials. We have termed these signals "isolated prepotentials" (IPPs) and interpret them to be discharges of the same presynaptic calyx terminal that produces the spike-preceding prepotentials (SPP).

Given the limitations of extracellular recording in vivo, there is no way to demonstrate with absolute certainty that IPPs and SPPs are the same signal and not discharges of different MNTB neurons or axons of passage in a multiunit recording. However, we feel that the bulk of evidence strongly supports the former interpretation: (1) During recording, IPPs and SPPs always appeared and disappeared in concert. If these signals originated from different sources, their "appearance" and "disappearance" during the recording should have frequently occurred independently of each other. (2) The principal component analysis showed that each of our unit recordings was composed of two, and only two, different types of waveforms: the pre\&action potential compounds and the IPP-EPSP complexes. If our recordings had been of the common multiunit type, the number of neuronal elements in different recordings, and consequently the number of different types of waveforms, would have varied between experiments. (3) In parallel studies of the paraolivary nucleus that employed the same experimental setup and identical type of selective, high-impedance electrodes (15-30 M $\Omega$ ) used here, only one signal type was observed in unit recordings. Multiunit activity was never recorded (unpublished observations). (4) The discharge pattern of SPPs and IPPs is inconsistent with the hypothesis that these signals arise from different independent neural elements. In response to highintensity tonal stimulation, IPPs exhibited a tonic firing pattern that lacked a prominent phasic component. In addition, the latency of the initial spike discharge was highly variable. To our knowledge, such a discharge pattern has never been described for MNTB neurons or globular bushy cells, which typically show primarylike responses or primarylike notch responses (e.g., Smith et al. 1991, 1998). In addition, during two-tone stimulation, IPPs and SPPs exhibited temporally correlated changes in spike number that cannot easily be accounted for if these signals are generated by different neural elements rather than by 

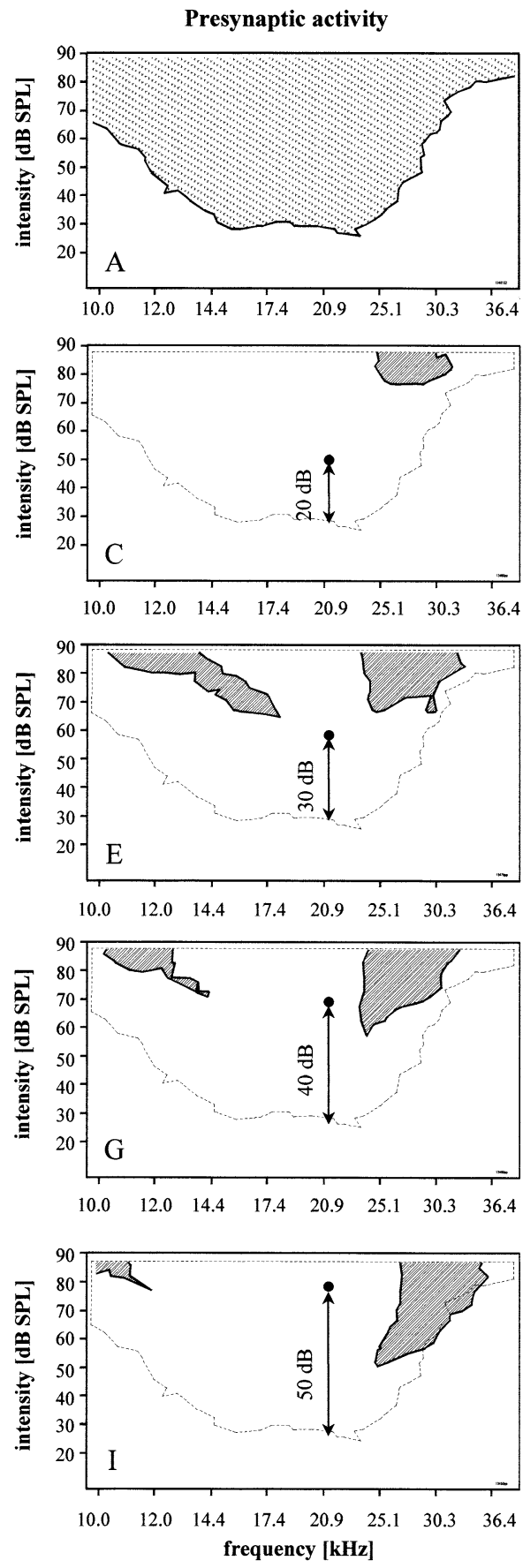

Postsynaptic activity
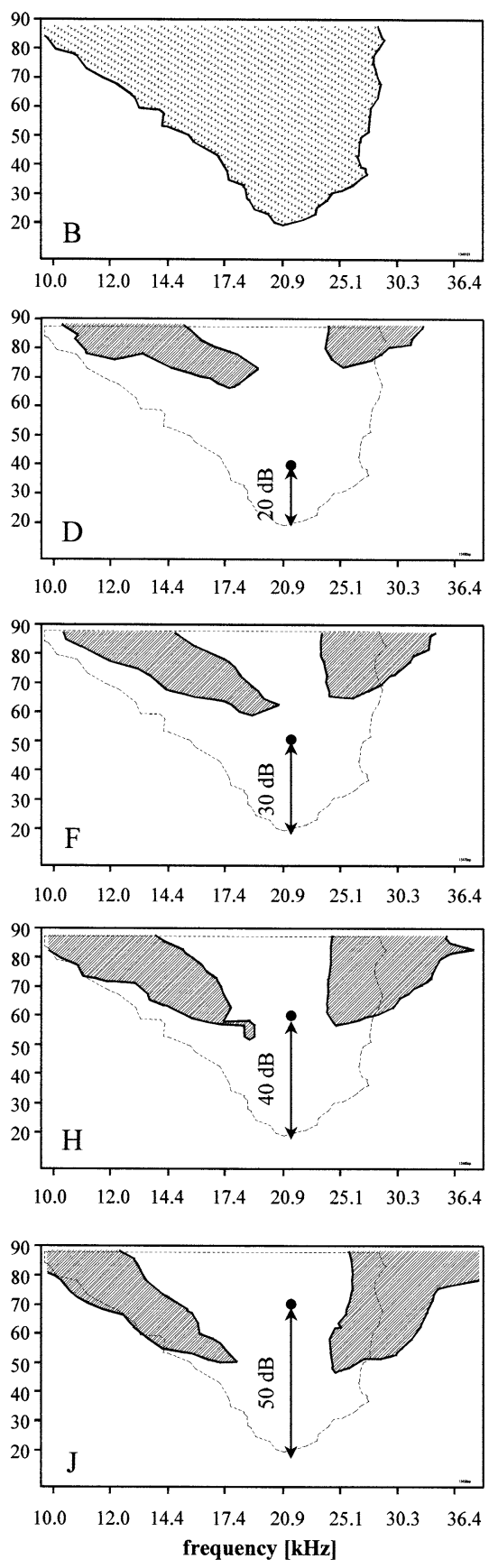

FIG. 17. Frequency tuning curves of presynaptic activity (left) and postsynaptic activity (right). Unit 13401. A, B. Area of suprathreshold excitatory response, determined with single-tone stimuli (100 ms duration). Note the narrower bandwidth in the postsynaptic activity (B) compared with the presynaptic activity (A). C-J. Inhibitory areas measured with twotone stimulation. The excitatory probe tones were presented at CF at different intensities above threshold as indicated in the respective graphs by closed circles. The tuning curves taken from $\mathbf{A}$ and $\mathbf{B}$ (dotted lines) are shown for comparison. Test-tones were randomly varied between 10 and $40 \mathrm{kHz}$ and 20 and $90 \mathrm{~dB}$ SPL. Hatched areas indicate frequency/intensity ranges in which the presentation of test-tones caused a significant reduction ( $\boldsymbol{p}=0.1$ ) of probetone-evoked discharge rates: Note that the reduction is more prominent for the postsynaptic activity (right column) compared with the presynaptic activity (left column) and covers a wider range of frequency/intensity pairs. the same calyx terminal (Fig. 14). (5) IPPs were observed only in recordings from units that also showed SPPs, i.e., in prepotential units.

Observations potentially inconsistent with the hypothesis that isolated prepotentials and spike-preceding potentials are the same signal There are two features in which the IPPs and the SPPs differ that are in contradiction to the assumption that both are generated by the same source: (1) the delayed negativity seen in connection with the IPPs and not with SPPs and (2) the differences in amplitudes of IPPs and SPPs.

Regarding the first, the recordings clearly show that the IPPs were typically followed after a slight delay by a negativity that peaked at $0.4-0.5 \mathrm{~ms}$. Such a negativity is not seen in the SPPs. We suggest that this negativity is an extracellularly recorded EPSP which in the SPPs is obscured by the postsynaptic action potential. Prepotentials in connection with postsynaptic EPSPs had been recorded in MNTB slice preparations by Borst et al. (1995) and by Chuhma and Ohmori (1998). In both studies the synaptic delay was 

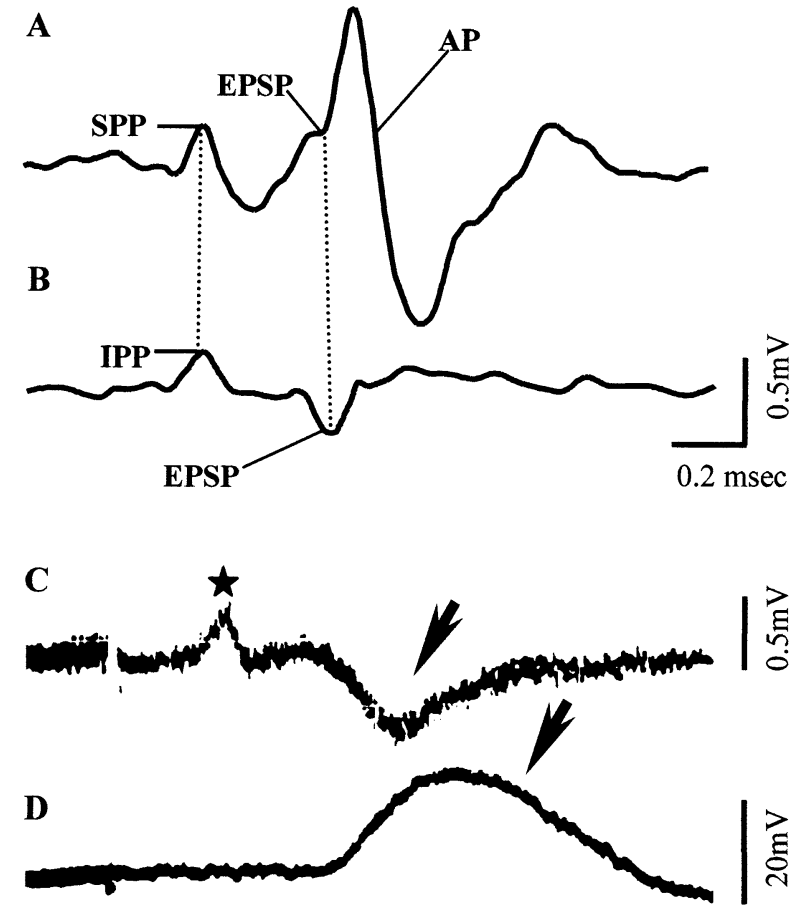

$1 \mathrm{msec}$

FIG. 18. Comparison of the waveforms of (A) preaction potential compounds and (B) IPP-EPSP complexes with simultaneous (C) extracellular and (D) intracellular recordings of pre- and postsynaptic activities (reported by Takeuchi and Takeuchi (1962)). A. Waveform of an extracellularly recorded preaction potential compound. B. IPPEPSP complex of an MNTB prepotential unit. C. Extracellular recording of the presynaptic axon of the giant synapse of Loligo. The presynaptic potential (star) is followed by a delayed negativity (arrow). D. Intracellularly recorded excitatory postsynaptic potential (arrow) occurs at the same time as the extracellular measured negativity. C and D reproduced from J. Gen. Physiol. 1962; 45:11811193 with copyright permission of The Rockefeller University Press.

comparable to the temporal relation between IPPs and subsequent negativity reported here. Support for our interpretation of the IPP-following negativity as an EPSP comes from a study by Takeuchi and Takeuchi (1962). They used a preparation of the Loligo giant synapse to simultaneously make extraand intracellular recordings of synaptic activity. The intracellularly recorded (positive) EPSP evoked by presynaptic electrical stimulation is extracellularly indicated by a negative voltage deflection which follows, with a brief delay, after the presynaptic spike (Fig. 18). Indeed, the compound IPP waveform we observed matches almost exactly (except the time domain) the extracellularly recorded signals described by Takeuchi and Takeuchi.

The second possible objection is not so easy to refute based on the present in vivo recordings. It is clear that in some units the SPPs and IPPs tended to differ in size, with the IPPs being somewhat smaller than the simultaneously recorded SPPs. The differences in amplitude may be attributable, in part, to the variability in signal size that characterized our extracellular recordings. The somewhat larger amplitude of the SPPs could also reflect a summation of excitatory afferent inputs. Based on Golgi studies, Morest (1969) reported that each MNTB neuron in the cat is contacted by a single calyx of Held. Subsequently, it was shown by the intracellular labeling of afferent fibers that a calyx terminal covers only about $24 \%$ of the somata of MNTB neurons in the cat (Smith et al. 1991). Immunolabeling, in contrast, revealed that up to $40 \%-48 \%$ of the soma surface of MNTB neurons is covered by excitatory terminals (Smith et al. 1991). This would suggest that at least a portion of the MNTB neurons may be contacted by noncalyceal excitatory terminals, possibly from collaterals of afferent fibers (Kuwabara et al. 1991). Consistent with this idea is the observation that sound stimulation in the cat can produce an activation of MNTB neurons that is mediated by noncalyceal excitatory inputs (Guinan and $\mathrm{Li}$ 1990). However, it remains questionable whether a summation of excitatory input through noncalyceal afferents accounts for the difference in signal size, because these small inputs would presumably not evoke synaptic potentials in MNTB neurons with the same short latency as the large calyceal inputs. Alternatively, differences in IPP and SPP amplitudes might be due to presynaptic effects of glycinergic inhibition (Turecek and Trussell 2001), a possibility that still requires experimental evidence from pharmacological in vivo studies. A third possibility is that the size difference between IPPs and SPPs is due to small random variations in the amplitude of presynaptic potentials. Then bigger presynaptic potentials would lead to more transmitter release, thereby producing bigger postsynaptic potentials and the occurrence of postsynaptic action potentials. This would turn the bigger presynaptic potentials into SPPs. By similar reasoning, the smaller presynaptic potentials would become IPPs.

\section{Failure of spike transmission}

The occurrence of prepotentials that were not followed by postsynaptic spikes indicates that signal transmission at the CN-MNTB synapse does not occur in a fixed one-to-one manner. Failure rates were relatively high, ranging from $52 \%$ and $45 \%$ for spontaneous and CF-driven activity, respectively, to $74 \%$ for two-tone stimulation.

Prior studies have also reported what appear to be failures in synaptic transmission. Guinan and $\mathrm{Li}$ (1990) found in their recordings from the cat MNTB that with electrical stimulation at high rates $(\geq 500$ 
$\mathrm{Hz}$ ), prepotentials were "sometimes" not followed by postsynaptic action potentials. Pfeiffer (1966), recording from spherical bushy cells in the cat AVCN, which also receive their excitatory afferent input from large calyceal endings, observed failures of postsynaptic spikes in about a quarter of the prepotential units studied. The presynaptic discharges associated with spike failures in these experiments could correspond to the IPPs in the present study. At the moment, we can only speculate about the causes of the higher incidence of spike failures that we observed. Possible reasons for this discrepancy might be species differences and differences in the depth or type of anesthesia between our respective studies.

The mechanism(s) responsible for the failure of presynaptic discharges to give rise to postsynaptic spikes could operate at the level of either the presynaptic terminal or the MNTB neuron itself. Possible mechanisms include the inhibition of spike discharges by neural inputs (neuronal inhibition) or a limitation of action potential firing due to the intrinsic membrane properties of the calyx terminal or the MNTB neuron. Rapidly activated 4-aminopyridine-sensitive potassium currents have been reported for MNTB neurons, and it is tempting to speculate that such currents might limit action potential generation (Forsythe and Barnes-Davies 1993a). However, the finding that failure rates increased dramatically during two-tone stimulation indicates that intrinsic membrane properties cannot be the only reason for failures in spike transmission. Under the conditions of two-tone stimulation, the failure of some prepotentials to give rise to postsynaptic spikes must have been mediated, at least in part, by an acoustically evoked inhibition acting at the calyx terminal and/or the postsynaptic neuron.

Presynaptic mechanisms. Presynaptic inhibition is one potential mechanism whereby sound stimulation could suppress the generation of postsynaptic spikes. GABA(B) receptors, known to be present in the membrane of MNTB calyx terminals, could mediate such an inhibitory effect through a G-protein-dependent decrease of presynaptic calcium permeability. This, in turn, would lead to a reduction in transmitter release (Isaacson 1998). This possibility is consistent with the reduction of excitatory postsynaptic potential (EPSC) amplitude following activation of GABA(B) receptors (Isaacson 1998). A comparable G-proteinmediated reduction of transmitter release could also result from autoactivation of presynaptic metabotrophic glutamate receptors, as has been reported for MNTB calyx terminals (Takahashi et al. 1996; von Gersdorff et al. 1997). However, both mechanisms would be too slow to account for the relatively rapid acoustically induced reduction in postsynaptic discharge activity observed in the present study.
Postsynaptic mechanisms. The sound-induced reduction of postsynaptic discharges is probably best accounted for by postsynaptic inhibition. Support for this interpretation comes from the finding that twotone stimulation caused large and immediate decreases in postsynaptic discharge rates without significantly affecting the level of presynaptic activity. This inhibitory influence could be mediated through the GABAergic and glycinergic inputs to MNTB somata that have been described in immunocytochemical and electronmicroscopical studies (Roberts and Ribak 1987; Adams and Mugnaini 1990; Cant 1991). Further evidence for postsynaptic inhibition and the functional role of these transmitters comes from slice electrophysiological studies (Banks and Smith 1992; Wu and Kelly 1995) and GABA (Wu and Kelly 1995). Both GABA and glycine caused a decrease in the input resistance of MNTB neurons, resulting in subthreshold EPSPs and the loss of action potential firing (Wu and Kelly 1995). In addition, long-latency IPSPs evoked by stimulation of the trapezoid body could be completely blocked by strychnine (Banks and Smith 1992).

\section{Possible contributions of inhibition to MNTB function}

Frequency selectivity. The presynaptic frequency selectivity of MNTB neurons was comparable to that of globular bushy cells, the source of excitatory afferent input to the MNTB (Rhode and Smith 1986). The properties of the inhibitory response areas measured under two-tone stimulation suggest that this pre- to postsynaptic increase in frequency selectivity is due to an acoustically evoked inhibition that prevents MNTB neuronal firing.

How does the sharpening of frequency selectivity of MNTB neurons relate to the activity of LSO neurons? The LSO and MNTB both receive their excitatory input from globular bushy cells within the VCN (Friauf and Ostwald 1988). LSO neurons, in turn, receive converging inhibitory inputs from the MNTB (Henkel and Gabriele 1999). This convergence of inputs results in a mismatch between excitatory and inhibitory tuning: At low stimulus levels an LSO neuron's inhibitory response area is more broadly tuned than its excitatory response field (Tsuchitani 1997). At higher intensity levels, however, the inhibitory tuning is sharpened so that this mismatch no longer exists. The sharpening of inhibitory tuning at higher intensities may well have its origin within the MNTB. Thus, the activation of sideband inhibition within the MNTB at higher intensities ( $>30 \mathrm{dBs}$ above threshold) results in an increase in frequency selectivity, which would be reflected in the inhibitory output to the LSO. 
Temporal response pattern. MNTB neurons showed phasic-tonic response patterns. Presynaptically, these could be classified as primarylike notch and postsynaptically as primarylike notch or primarylike. A primarylike notch pattern has also been described for the globular bushy cells, the source of afferent excitatory input to the MNTB and also for MNTB neurons themselves (Guinan et al. 1972b; Smith et al. 1991; Tsuchitani 1997). Pre- and postsynaptic activity differed in their temporal response characteristics. Specifically, the duration of the phasic component of the response was briefer postsynaptically and the overall level of discharge was reduced. Both effects might be attributable to acoustically evoked inhibition, which occurred with a slight delay relative to the onset of the excitatory response.

The functional significance of this delayed inhibition might be the enhancement of coding of fast amplitude fluctuations in complex acoustic signals. This could aid the detection of signals that barely stand out against background noise. A comparable suggestion was put forward by Ebert and Ostwald (1995) for the functionality of VCN neurons in rats based on observations of the effect of GABA on neuronal discharge activity.

Phase-locking fidelity. The present study showed phase-locked responses to low-frequency sinusoids in both presynaptic discharge activity and postsynaptic discharge activity. The goodness of phase-locking can be expressed by the entrainment of spikes and quantified by calculation of the synchronization index (Joris et al. 1994). The entrainment indicates the reliability with which a spike occurs during each stimulus cycle. Here we show that the entrainment is higher pre- than postsynaptically (Fig. 11). Comparable results were obtained by Wu and Kelly (1993) who used electrical stimulation in a slice preparation. However, despite the postsynaptic reduction in spike rates, the synchronization of the remaining spikes to a specific phase of the stimulus cycle (SI) is increased. Both the lower postsynaptic entrainment and the higher SIs indicate a possible influence of inhibition which causes presynaptic discharges occurring at unfavorable phases to fail to generate postsynaptic action potentials.

In conclusion, our results suggest that although MNTB cells receive a major excitatory input via calyces of Held, acoustically evoked inhibition also plays a principal role in MNTB signal processing. The failure of presynaptic discharges to generate postsynaptic action potentials results in the occurrence of IPPs. In a respective recording, IPPs and the SPPs are similar in a number of aspects which suggests that they originate from the same calyceal terminal. The differences between IPPs and SPPs described here might in turn account for their different efficiency to gen- erate postsynaptic action potentials. However, based on our current information, it is not possible to conclude that the failure of action potential generation at the CN-MNTB synapse is due only to postsynaptic inhibition. We should be able to verify the mechanisms underlying the failure of action potential generation at the CN-MNTB synapse by pharmacologically blocking the proposed inhibition.

\section{ACKNOWLEDGMENTS}

This research was founded by the Deutsche Forschungsgemeinschaft (GRK 250/1-96 and DFG Ru390-15/1). We acknowledge Gudrun König and Doris Freyberg for technical assistance. We thank Susanne Dehmel and Josh Gittleman for critical comments and many helpful discussions. Last but not least we are thankful to the two anonymous reviewers whose comments greatly improved this manuscript.

\section{REFERENCES}

Abeles M, Goldstein MH. Multispike train analysis. Proc. IEEE 65:762-773, 1977.

ADAMs JC. Heavy metal intensification of dab-based HRP reaction product [letter]. J. Histochem. Cytochem. 29:775, 1981.

Adams JC, Mugnaini E. Immunocytochemical evidence for inhibitory and disinhibitory circuits in the superior olive. Hear. Res. 49:281-298, 1990.

Arthur RM, Pfeiffer RR, SugA N. Properties of 'two-tone inhibition' in primary auditory neurones. J. Physiol. (Lond.) 212:593-609, 1971.

Banks MI, SMITH PH. Intracellular recordings from neurobiotinlabeled cells in brain slices of the rat medial nucleus of the trapezoid body. J. Neurosci. 12:2819-2837, 1992.

Bishop GH, Burke W, DAVIs R. Synapse discharge by single fibre in mammalian visual system. Nature 182:728-730, 1958.

Borst JG, Helmchen F, Sakmann B. Pre- and postsynaptic whole-cell recordings in the medial nucleus of the trapezoid body of the rat. J. Physiol. (Lond.) 489:825-840, 1995.

CANT NB. Projections to the lateral and medial olivary nuclei from the spherical and globular bushy cells of the anteroventral cochlear nucleus In: Altschuler BM, Clopton BM, BobBin RP, Hoffman DW (eds) The Central Auditory System Raven Press, New York, 1991, pp 99-119

Chumma N, Ohmori H. Postnatal development of phase-locked high-fidelity synaptic transmission in the medial nucleus of the trapezoid body of the rat. J. Neurosci. 18:512-520, 1998.

Cleland BG, Dubin MW, Levick WR. Simultaneous recording of input and output of lateral geniculate neurones. Nat. New Biol. 231:191-192, 1971.

Ebert U, Ostwald J. Gaba can improve acoustic contrast in the rat ventral cochlear nucleus. Exp. Brain Res. 104:310-322, 1995.

Forsythe ID, BARnES-DAVIES M. The binaural auditory pathway: Membrane currents limiting multiple action potential generation in the rat medial nucleus of the trapezoid body. Proc. $\mathrm{R}$ Soc. Lond. B. Bio. Sci. 251:143-150, 1993a.

Forsythe ID, BARnes-Davies M. The binaural auditory pathway: Excitatory amino acid receptors mediate dual timecourse excitatory postsynaptic currents in the rat medial nucleus of the trapezoid body. Proc. R. Soc. Lond. B Biol. Sci. 251:151-157, $1993 b$. 
Friauf E, Ostwald J. Divergent projections of physiologically characterized rat ventral cochlear nucleus neurons as shown by intra-axonal injection of horseradish peroxidase. Exp. Brain Res. 73:263-284, 1988.

GLASER EM, Ruchin DS. Principles of neurobiological signal analysis Academic Press, New York, 1976,

GOLDBERG JM, BROWN PB. Response of binaural neurons of dog superior olivary complex to dichotic tonal stimuli: Some physiological mechanisms of sound localization. J. Neurophysiol. 32:613-636, 1969.

Guinan Jr JJ, Guinan SS, Norris BE. Single auditory units in the superior olivary complex I: Responses to sounds and classification based on physiological properties. Int. J. Neurosci. 4:101120, 1972a.

Guinan JR JJ, Li RY. Signal processing in brainstem auditory neurons which receive giant endings (calyces of Held) in the medial nucleus of the trapezoid body of the cat. Hear. Res. 49:321-334, 1990.

Guinan Jr JJ, Norris BE, Guinan SS. Single auditory units in the superior olivary complex II: Locations of unit categories and tonotopic organization. Int. J. Neurosci. 4:b, 1972.

Helfert RH, Bonneau JM, Wenthold RJ, Altschuler RA. Gaba and glycine immunoreactivity in the guinea pig superior olivary complex. Brain Res. 501:269-286, 1989.

Henkel CK, Gabriele ML. Organization of the disynaptic pathway from the anteroventral cochlear nucleus to the lateral superior olivary nucleus in the ferret. Anat. Embryol. (Berl.) 199:149160, 1999.

Hubel DH, Wiesel TN. Integrative action in the cat's lateral geniculate body. J. Physiol. (Lond.) 155:385-398, 1961.

IRVINE DRF. The auditory brainstem Springer-Verlag, New York, 1986 ,

ISAACSON JS. GABA $\mathrm{b}_{\mathrm{b}}$ receptor-mediated modulation of presynaptic currents and excitatory transmission at a fast central synapse. J. Neurophysiol. 80:1571-1576, 1998.

Joris PX, Sмiтh PH, YIn TC. Enhancement of neural synchronization in the anteroventral cochlear nucleus. II. Responses in the tuning curve tail J. Neurophysiol. 71:1037-1051, 1994a.

KNIPSCHILD M, DöRRsCheIdT GJ, RÜBSAMEN R. Setting complex tasks to single units in the avian auditory forebrain. I: Processing of complex artificial stimuli. Hear. Res. 57:216-230, 1992.

Kopp C, Dörrscheidt GJ, Rübsamen R. Inhibition affects responsiveness of the medial nucleus of the trapezoid body in the gerbil Abstr. Assoc. Res. Otolaryngol. 643, 1997.

Kopp C, Dörrscheidt GJ, Rübsamen R. The MNTB is more than a relay. Abstr. Assoc. Res. Otolaryngol. 359, 1998.

Kunabara N, Dicaprio RA, Zook JM. Afferents to the medial nucleus of the trapezoid body and their collateral projections. J. Comp. Neurol. 314:684-706, 1991.

Kunabara N, Zook JM. Classification of the principal cells of the medial nucleus of the trapezoid body. J. Comp. Neurol. 314:707-720, 1991.

Lee BB, Virsu V, Creutzfeldt OD. Linear signal transmission from prepotentials to cells in the macaque lateral geniculate nucleus. Exp. Brain Res. 52:50-56, 1983.

MoREsT DK. The growth of synaptic endings in the mammalian brain: A study of the calyces of the trapezoid body. Z. Anat. Entwicklungsgesch. 127:201-220, 1968.

Osen KK, OTtersen OP. Glutamat receptors in the medial nucleus of the trapezoid body. International Symposium on Acoustical Signal Processing in the Central Nervous System, Prauge, September 4-7, 1996, Abstr. 70, 1996.
Ostapoff EM, Benson CG, Marie RL. GABA- and glycine-immunoreactive projections from the superior olivary complex to the cochlear nucleus in guinea pig. J. Comp. Neurol. 381:500-512, 1997.

Pfeiffer RR. Anteroventral cochlear nucleus: Wave forms of extracellularly recorded spike potentials. Science 154:667-668, 1966.

Rhode WS, Smith PH. Encoding timing and intensity in the ventral cochlear nucleus of the cat. J. Neurophysiol. 56:261-286, 1986.

Roberts RC, Ribak CE. GABAergic neurons and axon terminals in the brainstem auditory nuclei of the gerbil. J. Comp. Neurol. 258:267-280, 1987.

RYAn A. Hearing sensitivity of the Mongolian gerbil, meriones unguiculatis. J. Acoust. Soc. Am. 59:1222-1226, 1976.

SCHOfield BR. Projections to the cochlear nuclei from principal cells in the medial nucleus of the trapezoid body in guinea pigs. J. Comp. Neurol. 344:83-100, 1994.

SCHOfield BR, CANT NB. Organization of the superior olivary complex in the guinea pig. I. Cytoarchitecture, cytochrome oxidase histochemistry, and dendritic morphology J. Comp. Neurol. 314:645-670, 1991.

Smith PH, Joris PX, Carney LH, Yin TC. Projections of physiologically characterized globular bushy cell axons from the cochlear nucleus of the cat. J. Comp. Neurol. 304:387-407, 1991.

SMITH PH, Joris PX, YIN TC. Anatomy and physiology of principal cells of the medial nucleus of the trapezoid body (MNTB) of the cat. J. Neurophysiol. 79:3127-3142, 1998.

Sommer I, Lingenhohl K, Friauf E. Principal cells of the rat medial nucleus of the trapezoid body: An intracellular in vivo study of their physiology and morphology. Exp. Brain Res. 95:223-239, 1993.

Takahashi T, Forsythe ID, Tsujimoto T, Barnes-Davies M, Onodera K. Presynaptic calcium current modulation by a metabotropic glutamate receptor. Science 274:594-597, 1996.

TAKEUchi A, TAKeuchi N. Electrical changes in pre- and postsynaptic axons of the giant synapse of Loligo. J. Gen. Physiol. 45:1181-1193, 1962.

Tsuchitani C. Input from the medial nucleus of trapezoid body to an interaural level detector. Hear. Res. 105:211-224, 1997.

Turecer R, Trussell LO. Presynaptic glycine receptors enhance transmitter release at a mammalian central synapse. Nature 411:587-590, 2001.

Vahle-Hinz C, Hicks TP, Gottschaldt KM. Amino acids modify thalamo-cortical response transformation expressed by neurons of the ventrobasal complex. Brain Res. 637:139-155, 1994.

Gersdorff H, Schneggenburger R, Weis S, Neher E. Presynaptic depression at a calyx synapse: The small contribution of metabotropic glutamate receptors. J. Neurosci. 17:8137-8146, 1997.

Wu SH, Kelly JB. Synaptic pharmacology of the superior olivary complex studied in mouse brain slice. J. Neurosci. 12:30843097, 1992.

Wu SH, KELLY JB. Response of neurons in the lateral superior olive and medial nucleus of the trapezoid body to repetitive stimulation: Intracellular and extracellular recordings from mouse brain slice. Hear. Res. 68:189-201, 1993.

Wu SH, Kelly JB. Inhibition in the superior olivary complex: Pharmacological evidence from mouse brain slice. J. Neurophysiol. 73:256-269, 1995. 